\title{
GABA interneurons are the cellular trigger for ketamine's rapid antidepressant actions
}

\author{
Danielle M. Gerhard, ${ }^{1,2}$ Santosh Pothula, ${ }^{2}$ Rong-Jian Liu, ${ }^{2}$ Min Wu, ${ }^{2}$ Xiao-Yuan Li, ${ }^{2}$ Matthew J. Girgenti, ${ }^{2}$ Seth R. Taylor, ${ }^{2,3}$ \\ Catharine H. Duman, ${ }^{2}$ Eric Delpire, ${ }^{4}$ Marina Picciotto, ${ }^{2}$ Eric S. Wohleb, ${ }^{2,5}$ and Ronald S. Duman ${ }^{2}$

\begin{abstract}
'Department of Psychiatry, Weill Cornell Medicine, New York, New York, USA. ²Department of Psychiatry, Yale School of Medicine, New Haven, Connecticut, USA. ${ }^{3}$ Department of Cell and Developmental Biology, Vanderbilt University, Nashville, Tennessee, USA. ${ }^{4}$ Department of Anesthesiology, Vanderbilt University Medical School, Nashville, Tennessee, USA. ${ }^{5}$ Department of Pharmacology \& Systems
\end{abstract} \\ Physiology, University of Cincinnati College of Medicine, Cincinnati, Ohio, USA.
}

\begin{abstract}
A single subanesthetic dose of ketamine, an NMDA receptor (NMDAR) antagonist, produces rapid and sustained antidepressant actions in depressed patients, addressing a major unmet need for the treatment of mood disorders. Ketamine produces a rapid increase in extracellular glutamate and synaptic formation in the prefrontal cortex, but the initial cellular trigger that initiates this increase and ketamine's behavioral actions has not been identified. To address this question, we used a combination of viral shRNA and conditional mutation to produce cell-specific knockdown or deletion of a key NMDAR subunit, GluN2B, implicated in the actions of ketamine. The results demonstrated that the antidepressant actions of ketamine were blocked by GluN2B-NMDAR knockdown on CABA (Gad1) interneurons, as well as subtypes expressing somatostatin (Sst) or parvalbumin (Pvalb), but not glutamate principle neurons in the medial prefrontal cortex (mPFC). Further analysis of CABA subtypes showed that cell-specific knockdown or deletion of GluN2B in Sst interneurons blocked or occluded the antidepressant actions of ketamine and revealed sex-specific differences that are associated with excitatory postsynaptic currents on mPFC principle neurons. These findings demonstrate that GluN2B-NMDARs on GABA interneurons are the initial cellular trigger for the rapid antidepressant actions of ketamine and show sex-specific adaptive mechanisms to GluN2B modulation.
\end{abstract}

\section{Introduction}

The NMDA receptor (NMDAR) antagonist ketamine produces rapid-acting antidepressant effects in clinical studies of depressed patients and preclinical rodent models $(1,2)$. Although ketamine has been traditionally used as an anesthetic, more than a decade of research has been devoted to unraveling the mechanisms responsible for its rapid antidepressant effects. These efforts have uncovered a complex signaling cascade that results in activitydependent synapse formation and reversal of the synaptic deficits caused by chronic stress exposure (2-4). Early studies using a subanesthetic dose of ketamine found a rapid and transient increase in glutamate release in the medial prefrontal cortex (mPFC) (5). This glutamate burst is believed to initiate activity-dependent release of brain-derived neurotrophic factor and stimulation of protein synthesis-dependent synapse formation, both of which are required for the antidepressant behavioral actions of ketamine $(2,4)$. However, the initial cellular trigger for the rapid release of glutamate in the PFC and the antidepressant behavioral actions of ketamine remains unclear.

Two mechanistic hypotheses have been proposed for ketamine's initial cellular trigger $(6,7)$. The direct hypothesis posits

Authorship note: DMG and SP contributed equally to this work. Conflict of interest: The authors have declared that no conflict of interest exists. Copyright: ( 2020 , American Society for Clinical Investigation. Submitted: June 4, 2019; Accepted: November 13, 2019; Published: January 27, 2020 Reference information: J Clin Invest. 2020;130(3):1336-1349. https://doi.org/10.1172/JCI130808. that ketamine's antidepressant effects result from direct antagonism of NMDARs on principle-glutamatergic neurons, causing homeostatic synaptic plasticity and increased synaptic drive. Alternatively, the indirect hypothesis, or disinhibition hypothesis, proposes that ketamine first inhibits GABAergic interneurons, leading to the disinhibition of principle-glutamatergic neuron activity. During basal resting state conditions, pyramidal cell firing is inhibited by presynaptic tonic-firing GABA interneurons (8, 9). Because ketamine is an NMDAR open-channel blocker that requires activity to remove $\mathrm{Mg}^{2+}$ blocking entry to the channel, tonic-firing GABA interneurons should be more sensitive to low, antidepressant doses of ketamine, resulting in a rapid disinhibition of pyramidal neurons and the subsequent transient glutamate burst. Furthermore, as discussed in Homayoun and Moghaddam (10), fast-spiking GABAergic interneurons have faster excitatory postsynaptic potentials (EPSPs) than pyramidal neurons and are more effectively recruited by excitatory inputs $(11,12)$. These findings support earlier studies and suggest that NMDAR antagonists initially regulate the spontaneous firing of GABAergic interneurons to a greater extent than pyramidal neurons $(8,9)$. A recent study of hippocampal slices provides further support, demonstrating that ketamine reduces inhibitory input and disinhibits CA1 pyramidal neurons (13). Studies of GABA interneurons in depression have focused on the subtypes expressing somatostatin (Sst) or parvalbumin (Pvalb), which together make up approximately $70 \%$ of GABA interneurons (14). These 2 subtypes innervate different aspects of excitatory neurons (Sst dendrites, Pvalb cell bodies) and have different electrophysiological properties (15). 
A

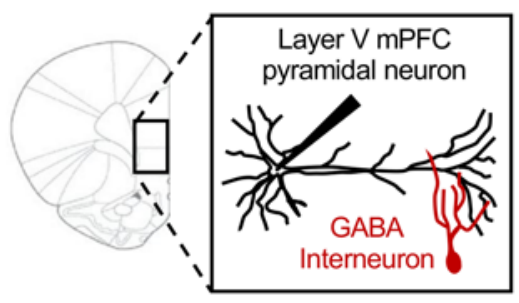

B

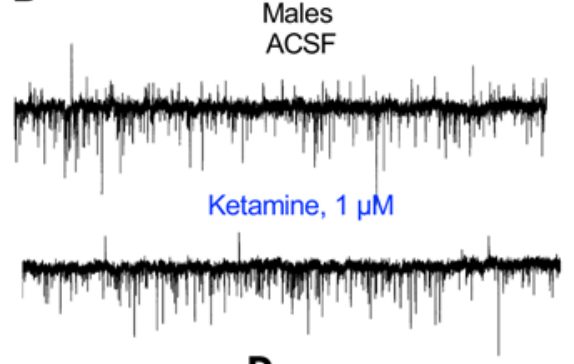

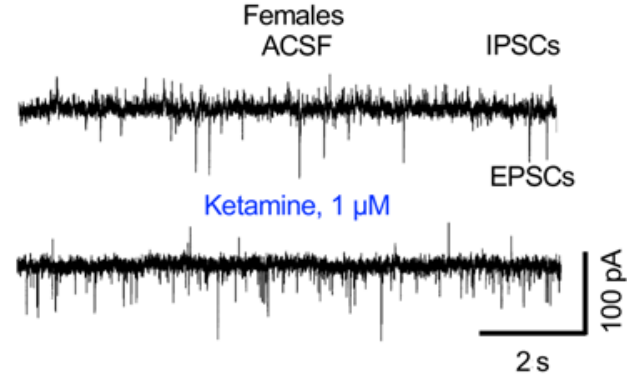

$2 \mathrm{~s}$
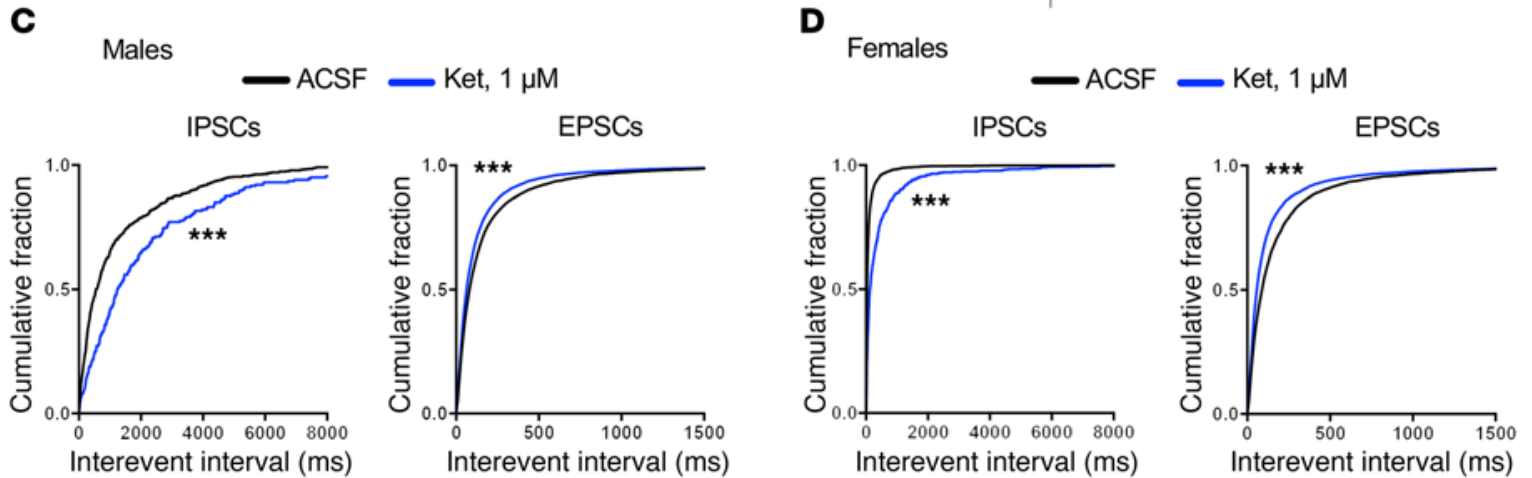

Figure 1. Ketamine reduces spontaneous inhibitory postsynaptic currents in mPFC slices. (A) Brain slice electrophysiology schematic. (B) Representative traces of spontaneous inhibitory postsynaptic currents (sIPSCs) and spontaneous excitatory postsynaptic currents (sEPSCs) from male and female mice before and after application of $1 \mu \mathrm{M}$ ketamine. (C) In male mice, $1 \mu \mathrm{M}$ ketamine significantly decreased sIPSCs and increased sEPSCs ( $n=9-10$ cells, 10 mice, $\left.{ }^{* * *} P<0.001\right)$. (D) In female mice, $1 \mu \mathrm{M}$ ketamine significantly decreased sIPSCs and increased sEPSCs $\left(n=7-8\right.$ cells, 5 mice, $\left.{ }^{* * *} P<0.001\right)$. Kolmogorov-Smirnov 2-sample test was used. Data represented as the cumulative probability of the interevent interval. Abbreviations: ACSF, artificial cerebrospinal fluid; Ket, ketamine.

NMDARs are a heterotetramer complex composed of 2 obligatory GluN1 subunits and 2 additional subunits that are a combination of GluN2A, GluN2B, GluN2C, or GluN2D. Previous studies have demonstrated that a selective GluN2B-negative allosteric modulator (Ro 25-6981) produces antidepressant behavioral effects and synaptic changes similar to ketamine in rodent models $(2,3,16)$. In addition, clinical studies have demonstrated that a single dose of another selective GluN2B-negative allosteric modulator, CP-101,606, produces antidepressant actions in depressed patients (17). Here, we used a virus-mediated shRNA knockdown strategy and conditional deletion approach to target GluN2B subunits on different populations of cells in the mPFC, and thereby determine their role in mediating ketamine's antidepressant behavioral effects. We combined electrophysiological, behavioral, and molecular techniques to determine whether GluN2B-containing NMDARs on excitatory neurons or GABAergic interneurons in the $\mathrm{MPFC}$ are necessary for ketamine's antidepressant effects.

\section{Results}

Ketamine reduces inhibitory postsynaptic currents in the $M P F C$. We first tested the influence of ketamine on spontaneous postsynaptic currents in layer $\mathrm{V}$ pyramidal neurons of the MPFC (Figure 1). If ketamine preferentially acts at NMDARs on GABAergic interneurons, we would expect a reduction in the inhibitory tone or frequency of spontaneous inhibitory postsynaptic currents (sIPSCs). In order to record sIPSCs and spontaneous excitatory postsynaptic currents (sEPSCs) simultaneously, whole-cell patch-clamp recordings were performed at a holding potential of $-65 \mathrm{mV}$ (the
$\mathrm{Cl}^{-}$reversal potential in the pipette solution was $-72 \mathrm{mV}$ ). Spontaneous postsynaptic currents were determined before and during bath application of ketamine at $1 \mu \mathrm{M}$, the estimated concentration reached in the human brain after i.v. infusion of a dose in the therapeutic range (18) and a concentration that blocks approximately $50 \%$ of NMDA-induced currents (19); or $10 \mu \mathrm{M}$, a concentration that is sufficient to block approximately $80 \%$ of NMDA-induced currents (20). These concentrations have also been used in recent reports $(4,13)$. The results demonstrated that $1 \mu \mathrm{M}$ ketamine significantly decreased sIPSC, but increased sEPSC in male and female mice (Figure 1, A-D); the cumulative probability curves for interevent intervals were shifted to the right for sIPSCs (i.e., longer intervals, decreased frequency), whereas for sEPSCs the curves were shifted to the left (i.e., shorter intervals, increased frequency) (Figure 1, C and D). Ketamine at $1 \mu \mathrm{M}$ increased sEPSC amplitude in male and female mice, corresponding to increased sEPSC frequency; sIPSC amplitude was increased in males but decreased in females (the reason for this difference is unknown) (Supplemental Figure 1, E and F; supplemental material available online with this article; https://doi.org/10.1172/JCI130808DS1). In contrast, $10 \mu \mathrm{M}$ ketamine significantly decreased basal rates of sIPSCs and sEPSCs in male and female mice (Supplemental Figure 1, A-D). These findings are consistent with the hypothesis that GABA interneuron-mediated IPSCs are more sensitive to ketamine blockade than glutamate pyramidal neuron-mediated EPSC responses.

Knockdown of NMDAR-GluN2B on GABA but not glutamate neurons produces baseline antidepressant-like effects and occludes the 


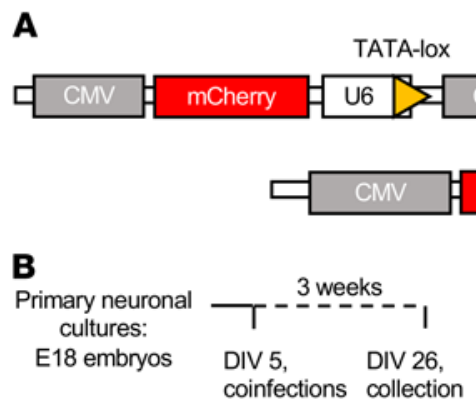

E

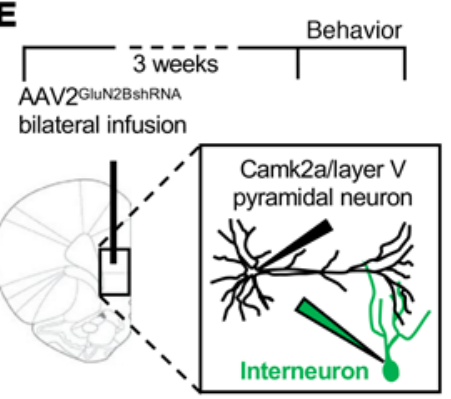

$\mathbf{F}$

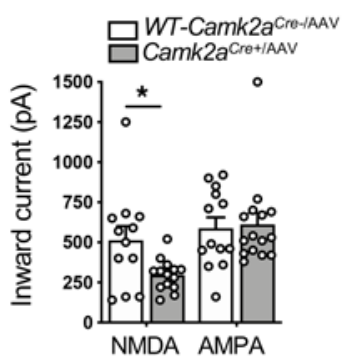

G

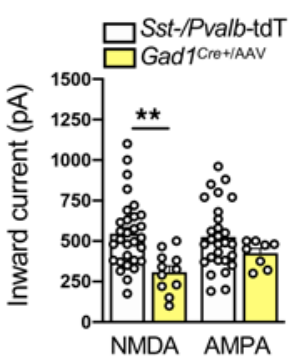

H

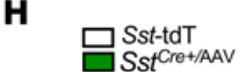

I
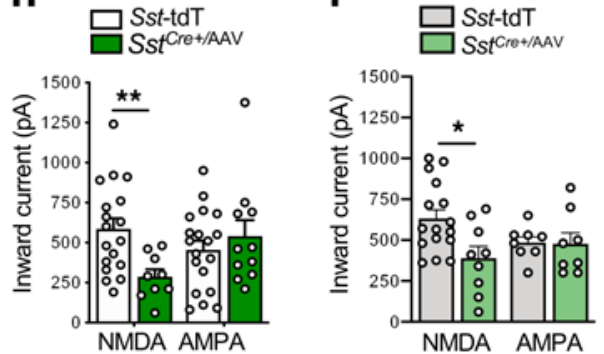

J
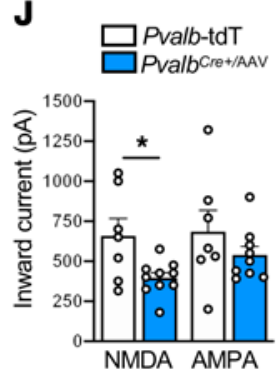

Figure 2. AAV2 ${ }^{\text {Clun2BshRNA }}$ knocks down GluN2B and reduces NMDA inward currents in a

Cre-dependent manner. (A) Schematic of the pGluN2BshRNA knockdown construct before and after introduction of Cre recombinase to generate the active construct. (B) Schedule for cell culture experiments. (C) There was a significant reduction in GluN2B protein in the AAV2 ${ }^{\text {CluN2BshRNA }}+$ AAV1-Cre (AAV2-Cre) group compared with AAV2 ${ }^{\text {Clunz2BshRNA }}$ (AAV2) or AAV2-Cre alone (Cre) ( $n=5$ per group, 1-way ANOVA with Tukey's multiple-comparisons test, $\left.\left[F_{2,12}=14.1, P<0.001\right]\right)$. (D) Representative images of Western blots. Lanes were run on the same gel and were contiguous. (E) Schematic of the schedule for brain slice electrophysiology experiments. (F-J) The influence of AAV2 $2^{\text {Clun2BshRNA }}$ on NMDAR and AMPAR inward currents was tested across genotypes. There was a significant reduction in NMDA-induced inward currents in all genotypes compared with their controls. Unpaired 2 -tailed $t$ tests were performed. (F) Camk2a $a^{\text {Cret/AAV }}$ (Camk2a $a^{\text {Cret/AAv: }} n=3$ mice, 14-15 cells; WTCamk2 $a^{\text {Cret/AAv}}: n=2$ mice, $12-13$ cells; $\mathrm{t}_{24}=2.510$,

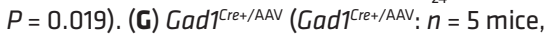
8-11 cells; Sst-/Pvalb-Td-Tomato [TdT]: $n=10$ mice, $28-30$ cells; $\left.t_{39}=3.447, P=0.0014\right)$. (H) Sst ${ }^{\text {Cret } / \text { AAV }}$ males (Sst $t^{\text {Cret/AAV: }} n=2$ mice, 9-11 cells; Sst-TdT: $n=8$ mice, 16-17 cells; $\mathrm{t}_{25}=2.976, P=$ 0.006). (I) Sst $t^{\mathrm{Cr}+/ \mathrm{AAV}}$ females (Sst $t^{\text {Cret/AAv }}: n=3$ mice, $8-9$ cells; Sst $-\mathrm{TdT}: n=9$ mice, $8-16$ cells; $\mathrm{t}_{23}=$ 2.686, $P=0.013$ ). (J) Pvalb $b^{\text {Cret/AAV }}$ males ( $P$ valb $b^{\text {Cret/AAv: }}$ : $n=3$ mice, 9-10 cells; Pvalb-TdT: $n=5$ mice, 7 cells; $\left.\mathrm{t}_{15}=2.696, P=0.017\right)$. There were no significant differences in AMPA-induced inward currents in any genotype. ${ }^{*} P<0.05 ;{ }^{* *} P<0.01 ;{ }^{* * *} P<$ 0.001 . All data are represented as mean \pm SEM. Abbreviations: tdT, td-Tomato. actions of ketamine. First, we confirmed that glutamatergic pyramidal neurons and GABAergic interneuron subtypes in the MPFC express GluN2B using immunohistochemistry: GluN2B colabeled with CaMKII (pyramidal cell) or glutamate decarboxylase 67 (GAD67; GABA interneuron) cells (labeled using specific antibodies) and Sst- or Pvalb-tdTomato ${ }^{+}$cells (Supplemental Figure 2, A-D). Furthermore, bath application of the selective GluN2B antagonist conantokin $\mathrm{G}(2 \mu \mathrm{M})$ reduced the NMDA-induced inward currents in pyramidal, Sst, and Pvalb cells in the MPFC (Supplemental Figure 3), demonstrating the expression of GluN2B subunits.

To target specific cell types, we used an shRNA-mediated adeno-associated virus (AAV) cell-type specific knockdown (sMACK-down) approach, combining viral expression of shRNA targeting GluN2B with cell-specific Cre recombinase (Cre ${ }^{+}$) transgenic mouse lines. The GluN2BshRNA construct (Figure 2A) expressed both mCherry and EGFP, and in the presence of Cre recombinase, EGFP was deleted, resulting in expression of only mCherry (as a marker of recombination) and GluN2BshRNA. The GluN2BshRNA construct was packaged into an adeno-

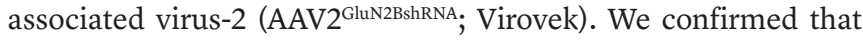
virus-mediated GluN2BshRNA expression reduced GluN2B in a Cre-dependent manner in primary neuronal cultures (Figure 2, B-D). Additionally, we confirmed that cell-specific virus-mediated knockdown of GluN2B significantly reduced the NMDA- but not AMPA-induced inward currents in layer $\mathrm{V}$ pyramidal neurons in different lines of Cre recombinase-transgenic mice used in this study, including Camk2a-Cre (Camk2a $\left.a^{\mathrm{Cre}+}\right), \mathrm{Gad}^{\mathrm{Cre+}}, \mathrm{Sst}^{\mathrm{Cre+}}$, and $\mathrm{Pvalb}^{\mathrm{Cre+}}$ male mice and in $S s t^{\mathrm{Cre}+}$ female mice (Figure 2, E-J). To identify interneurons for the slice electrophysiology experiments, Sst-Td-Tomato mice (for Sst ${ }^{\text {Cre+ }}$ ), Pvalb-Td-Tomato mice (for Pvalb ${ }^{\text {Cret }}$ ), or both Pvalb-Td-Tomato and Sst-Td-Tomato mice (for Gad1 $1^{\text {Cre+ }}$ ) were used as controls.

Initial behavioral studies were conducted in Camk $2 a^{\text {Cre+ }}$ mice to target principle neurons and in $\mathrm{Gad}^{\mathrm{Cre+}}$ mice to target all GABA interneuron subtypes. We also focused on male mice with a plan to extend the results to females after we identified which cell type was involved in the ketamine response. AAV2 ${ }^{\text {Glun2BshRNA }}$ was infused bilaterally into the $\mathrm{MPFC}$ (prelimbic and infralimbic 
regions) of Camk2a $a^{\text {Cre+ }}$ and Gad1 ${ }^{\text {Cret }}$ adult (8-12 week) male mice and WT Camk2a $a^{\text {Cre- }}$ and Gad1 ${ }^{\text {Cre- }}$ littermate controls (Figure 3A). The MPFC was targeted because prior studies have shown that this region is necessary and sufficient for the antidepressant actions of ketamine $(2,21)$. Three weeks after viral infusion to allow for GluN2BshRNA expression and GluN2B knockdown, the mice underwent behavioral testing, and then brains were collected for

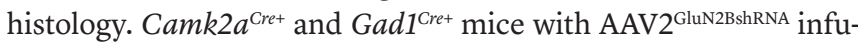
sions (referred to as Camk2 $a^{\mathrm{Cre}+\mathrm{AAV}}$ and $\mathrm{Gad} 1^{\mathrm{Cre+} / \mathrm{AAV}}$, respectively) displayed different subsets of cells with mCherry only (Figure 3, $\mathrm{B}$ and $\mathrm{F}$ ), demonstrating cell-specific recombination and expression of the GluN2BshRNA construct in each transgenic line. In WT-Camk $2 a^{\mathrm{Cre}-/ \mathrm{AAV}}$ and WT-Gad1 ${ }^{\mathrm{Cre}-/ \mathrm{AAV}}$ controls, the labeled neurons were yellow (i.e., both mCherry and EGFP), demonstrating that there was no recombination as expected.

Baseline testing was conducted to evaluate immobility, referred to as preswim (before ketamine) as part of the forced swim test (FST, a measure of behavioral despair); in addition, preswim is often used as an initial stressor before testing antidepressants in this paradigm. Baseline behavior was also analyzed in an open field test (OFT), a measure of ambulatory activity (distance traveled) and anxiety behavior (time spent in center); the OFT also serves as an additional mild stressor prior to ketamine treatment. One day after the OFT, mice were administered vehicle (saline, i.p.) or a single dose of ketamine $(10 \mathrm{mg} / \mathrm{kg}$, i.p.) and then tested 1 and 3 days later in the FST and novelty-suppressed feeding test (NSFT, a measure of anxiety-like behavior), respectively. This dose of ketamine produces a rapid transient burst of glutamate and antidepressant behavioral responses $(2-5,15,19)$. The behavioral testing times ( 1 and 3 days) were used to avoid the acute locomotor and sensorimotor effects of ketamine administration and still show the rapid and sustained antidepressant effects of ketamine (Figure 3A). In Camk2 $a^{\mathrm{Cre}+\mathrm{AAV}}$ male mice, there were no differences in preswim immobility, or on center time or distance in the OFT (Figure 3C). Ketamine significantly decreased immobility in the FST in WT-Camk $2 a^{\mathrm{Cre}-/ \mathrm{AAV}}$ and Camk $2 a^{\mathrm{Cre}+/ \mathrm{AAV}}$ mice (Figure $3 \mathrm{D})$. In the NSFT, ketamine administration significantly reduced the latency to feed in WT-Camk $2 a^{\mathrm{Cr}-/ \mathrm{AAV}}$ and Camk $2 a^{\mathrm{Cre}+/ \mathrm{AAV}}$ mice (Figure $3 \mathrm{E}$ ). No significant differences were observed in home cage feeding (Figure 3E).

Using the same design, we found that $G a d 1^{\mathrm{Cret} / \mathrm{AAV}}$ mice displayed significantly decreased baseline immobility in the preswim test, an antidepressant-like effect, compared with WT-Gad $1^{\text {Cre-AAV }}$ controls (Figure 3G); there were no significant effects on baseline behaviors in the OFT center time or total distance (Figure $3 G)$. Saline-treated Gad1 $1^{\mathrm{Cre} / \mathrm{AAV}}$ mice showed a significant baseline effect, similar to the pretest, and ketamine administration had no further effect beyond the baseline changes (Figure $3 \mathrm{H}$ ). In WT-Gad1 ${ }^{\text {Cre-AAV }}$ controls, ketamine administration significantly decreased FST immobility time (Figure $3 \mathrm{H}$ ). In the NSFT, ketamine treatment significantly reduced latency to feed compared with saline in the WT-Gad1 $1_{\text {Cre-/AAV }}$ controls (Figure $3 \mathrm{I}$ ). In Gad1 $1^{\mathrm{Cre}+/ \mathrm{AAV}}$ mice, there was a nonsignificant decrease in baseline latency to feed, and the actions of ketamine were blocked or occluded (Figure 3I). No significant differences were observed in home cage feeding (Figure 3I). To address the possibility that GluN2B knockdown could produce a floor effect on immobility, we treated a separate cohort of Gad1 ${ }^{\mathrm{Cre+} / \mathrm{AAV}}$ male mice with $20 \mathrm{mg} / \mathrm{kg}$ desipramine (DMI), a norepinephrine-selective reuptake inhibitor (22). DMI administration significantly reduced immobility in the FST in WT-Gad1 $1^{\mathrm{Cre} / \mathrm{AAV}}$ and Gad1 $1^{\mathrm{Cre}+\mathrm{AAV}}$ mice (Supplemental Figure 4).

Knockdown of GluN2B in Sst interneurons produces sex-specific baseline behavioral effects and blocks the antidepressant-like effects of ketamine. To extend the results of the Gad1 ${ }^{\text {Cret }}$ study, we investigated the role of 2 major GABAergic interneuron subtypes, Sst and Pvalb, in the rapid antidepressant actions of ketamine (23). Sst and Pvalb interneurons display different molecular, electrophysiological, morphological, and circuit connectivity properties that could differentially contribute to the actions of ketamine (23). Postmortem studies of depressed subjects and rodent studies of chronic stress highlight a selective vulnerability of Sst-expressing GABAergic interneurons in PFC subregions $(24,25)$. We have reported that the initial cellular trigger for another rapid-acting antidepressant, scopolamine, is dependent on Sst but not Pvalb interneuron M1-type muscarinic acetylcholine receptors (26). Based on these findings, we initiated studies of Sst interneurons, including virus-mediated shRNA GluN2B knockdown and devel-

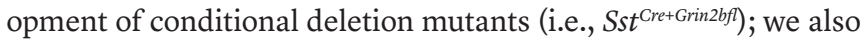
compared male and female mice and conducted electrophysiological and RNA-Seq studies.

Electrophysiology studies have demonstrated that Sst interneurons are responsive to NMDA, and that this effect is blocked by a selective GluN2B antagonist, conantokin G (Supplemental Figure 3); immunohistochemistry studies have demonstrated that $S s t^{+}$interneurons in the mPFC express GluN2B (Supplemental Figure 2, C and D). Sst GluN2B knockdown studies were carried out as described above. Sst ${ }^{\text {Cret }}$ mice were infused with AAV2 ${ }^{\text {Glun2BshRNA }}$ ( $\left.S s t^{\mathrm{Cre}+\mathrm{AAV}}\right)$ into the $\mathrm{mPFC}$, resulting in cell type-specific recombination (mCherry only, red cells), compared with WT-Sst Cre-/AAV control mice with no recombination (mCherry + EGFP double-labeled yellow cells) (Figure 4, A and B). Functional knockdown was also shown in $S s t^{\mathrm{Cret} / \mathrm{AAV}}$ male and female mice by slice physiology, with decreased NMDA- but not AMPA-induced inward currents (Figure 2, $\mathrm{H}$ and I). In male $S s t^{\mathrm{Cre} / \mathrm{AAv}}$ mice, there was a significant decrease in immobility time in the preswim test compared with $W T$-Sst $t^{\mathrm{Cre} / \mathrm{AAV}}$ controls (Figure $4 \mathrm{C}$ ); however, in female $S s t^{\mathrm{Cret} / \mathrm{AAV}}$ mice, there was no significant effect on immobility time (Figure $4 \mathrm{C}$ ). There were no effects in male or female $S s t^{\mathrm{Cret} / \mathrm{AAV}}$ mice in the OFT (Figure 4, D and E). A single dose of ketamine significantly decreased FST immobility time in male and female WT-Sst ${ }^{\text {Cre-/AAV }}$ mice compared with saline controls (Figure 4, F and I). In saline-treated $S s t^{\mathrm{Cre}+\mathrm{AAV}}$ mice, there was a significant decrease in baseline FST immobility in males and a trend for a decrease in females, and ketamine administration had no further effects in either sex (Figure 4, F and I). In the NSFT, ketamine significantly reduced latency to feed in male and female WT-Sst $t^{\text {Cre-/AAv }}$ mice when compared with saline controls, but these effects were completely absent in $S s t^{\mathrm{Cre}+\mathrm{AAv}}$ mice (Figure 4, G and J). There was a small decrease in baseline latency to feed in female $S s t^{\mathrm{Cre}+/ \mathrm{AAV}}$ mice, although this effect was not significant. No significant differences were observed in home cage feeding (Figure 4, G and J). In males, we also examined a reward-seeking behavior, the female urine sniffing test (FUST) (27). Male WT-Sst ${ }^{\text {Cre-/AAV }}$ mice administered ketamine spent significantly more time sniffing female urine 
$\mathbf{A}$

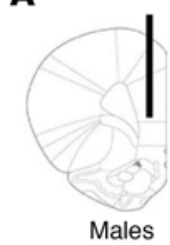

AAV2Glun2BshRNA

bilateral infusion,

WT and Cre+ lines

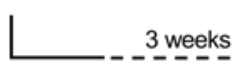

3 weeks

$-\frac{-2}{1}-1$

Saline or ketamine (10 mg/kg i.p.)
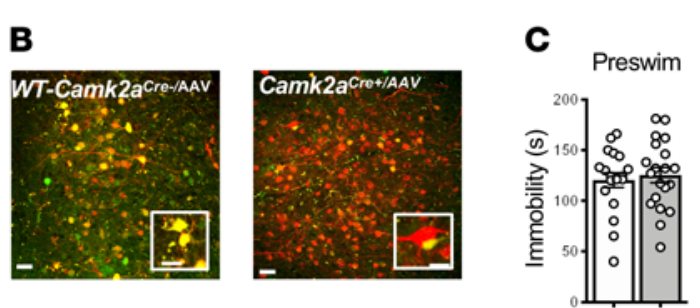

E

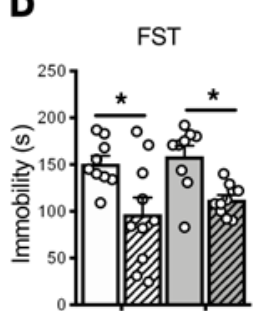

NSFT
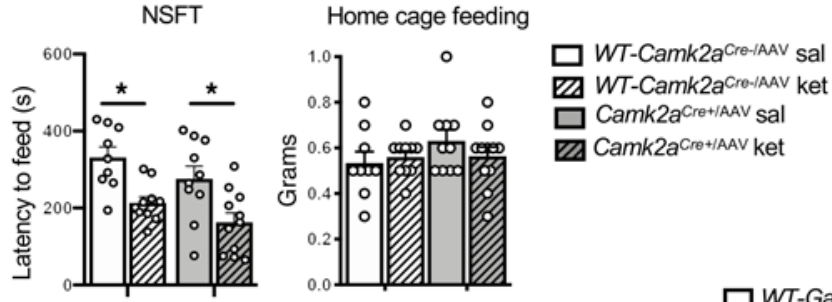

$\mathbf{F}$
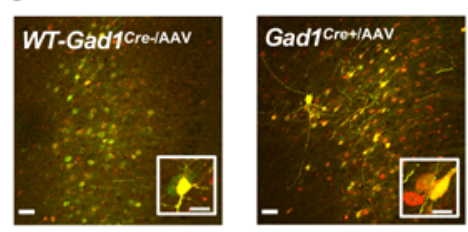

\section{G}
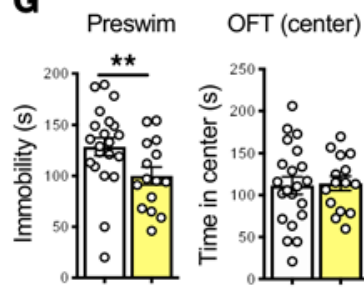

OFT (distance)

$\square$ Gad1Cro+/AAV

H

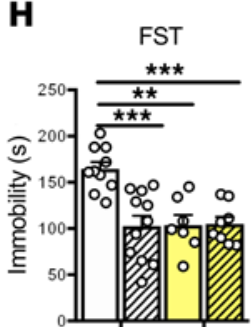

I

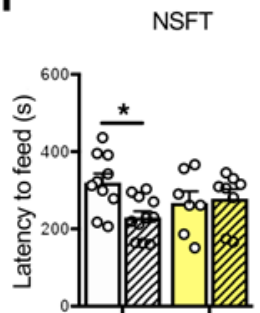

Home cage feeding

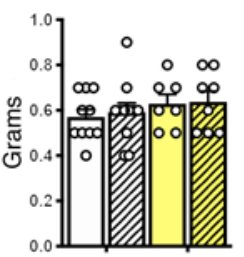

Figure 3. Infusion of AAV2 $2^{\text {CluN2BshRNA into }}$ mPFC of $\mathrm{Cad1}^{\mathrm{Cre+}}$, but not Camk $2 a^{\mathrm{Cre}+}$, male mice occludes the antidepressant effects of ketamine. (A) Procedure schematic. (B and F) Representative images of AAV2 $2^{\text {CluN2BshRNA }}$ mediated expression and recombination in the MPFC of (B) WT-Camk2a Cre-, Camk2a $a^{\mathrm{Cre+}}$, (F) WT-Gad1 ${ }^{\text {Cre-/AAV }}$, and Gad1 $7^{\text {Cre+/AAV }}$ male mice. Scale bars: $50 \mu \mathrm{m}$ and $20 \mu \mathrm{m}$ (insets). (C) In Camk2a $a^{\text {Cre+/AAV }}$ mice, there was no effect of GluN2B knockdown on baseline immobility (preswim) or time spent in center and distance traveled in the open field test (OFT; $n=$ 18-21 per group). (D) WT-Camk2a Cre-/AAV-ket and Camk2 $a^{\text {Cre+/AAV }}$-ket mice showed significantly reduced immobility in the forced swim test (FST) compared with saline controls (n = 12-14 per group, treatment: $F_{1,34}=18.18$, $P=0.0002$ ). (E) WT-Camk2a $a^{\text {Cre- } / \text { AAV }}-$ ket and Camk2a 2 Cre+/AAV $-k e t$ mice showed significantly reduced latency to feed in the noveltysuppressed feeding test (NSFT; $n=9-11$ per group, treatment: $\mathrm{F}_{1,36}=19.89, P<0.0001$, genotype: $\left.F_{1,36}=4.186, P=0.0481\right)$. No significant differences were found in home cage feeding. (G) There was a significant reduction in preswim immobility in Gad7 $7^{\text {Cre+/AAV }}$ mice, but no effect on time spent in center and distance traveled in the OFT ( $n=15-21$ per group, $\left.\mathrm{t}_{34}=2.226, P=0.0327\right)$. (H) Only

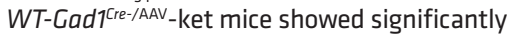
reduced immobility in FST compared with their saline controls ( $n=7-11$ per group, genotype: $\mathrm{F}_{1,32}=8.662, P=0.006$, treatment: $\mathrm{F}_{1,32}=9.313, P=0.0045$ genotype times treatment: $\mathrm{F}_{1,32}=10.04, P=0.0034$ ). (I) Only WT-Gad1 ${ }^{\text {Cre-/AAV }}$-ket mice showed significantly reduced latency to feed in the NSFT ( $n=$ 7-11 per group, genotype times treatment: $\left.\mathrm{F}_{1,32}=4.716, P=0.0374\right)$. Preswim and OFT: unpaired 2-tailed $t$ test. FST and NSFT: 2-way ANOVA with Tukey's multiple-comparisons test. ${ }^{*} P<0.05$; ${ }^{* *} P<0.01$; ${ }^{* *} P<0.001$. All data are represented as mean \pm SEM. Abbreviations: sal, saline; ket, ketamine. when compared with their genotype controls, and this effect was blocked in $S s t^{\mathrm{Cre}+\mathrm{AAV}}$ mice (Figure $4 \mathrm{H}$ ). There was no effect on baseline FUST in male $S s t^{\mathrm{Cre}+\mathrm{AAV}}$ mice, and no significant differences in time sniffing water across any group (Figure $4 \mathrm{H}$ ).

To examine possible cellular determinants of the baseline behavioral differences in male and female $S s t^{\mathrm{Cret} / \mathrm{AAV}}$ mice, we measured sIPSCs and sEPSCs in layer $\mathrm{V}$ pyramidal neurons in mPFC slices (Figure 4, $\mathrm{K}$ and L). In $S s t^{\mathrm{Cret} / \mathrm{AAV}}$ males, there was a small but significant decrease in the frequency of sIPSCs, but a larger increase in sEPSCs (Figure $4 \mathrm{~K}$ ). In female $S s t^{{ }^{\mathrm{Cre}+} / \mathrm{AAV}}$ mice, there was a significant decrease in the frequency of sIPSCs, but no effect on sEPSCs (Figure $4 \mathrm{~L}$ ). The increase in basal sEPSCs in male mice could contribute to the baseline antidepressant-like effects observed in the preswim in male versus female $S s t^{\mathrm{Cre}+/ \mathrm{AAV}}$ knockdown mice (Figure 4C). Despite there being no significant difference in sEPSCs in female mice, there was still an occlusion of ketamine's effects in female $S s t^{\mathrm{Cre}+\mathrm{AAV}}$ knockdown mice. These findings suggest that there may be varying molecular and cellular profiles that account for the observed sex differences.

To further examine cellular differences between males and females, we examined NMDAR subunit composition in Sst interneurons. Sst-tdTomato interneurons from mPFC were sorted by FACS and levels of NMDAR subunits determined by RNA-Seq. We found that expression of the GluN2B subunit, encoded by the Grin $2 b$ gene, in the male and female $\mathrm{mPFC}$ is higher compared with Grin $2 a$, similar to reports of Grin $2 b$ predominance in $S s t^{+}$interneurons of the adult male mouse visual cortex (28). In addition, there were no significant sex-specific differences in the expression of Grin1, Grin2a, and Grin2b in Sst interneurons (Supplemental Figure 5, A and B). Because NMDAR channel opening 
requires AMPA receptor (AMPAR) activity, we also examined the relative abundance of AMPAR subunits 1 and 2, encoded by the Gria1 and Gria2 genes, respectively. Here, we observed significantly higher levels of Gria2 and a trend for higher levels of Gria1 in female mice compared with male mice (Supplemental Figure $5 \mathrm{C}$ ). Further studies are required to determine whether differences in AMPAR expression contribute to the behavioral differences in males and females and whether GluN2B knockdown alters the expression patterns of NMDAR and AMPAR subunits.

Because ketamine is a nonselective NMDAR channel blocker, we tested whether knockdown of another subunit, GluN2A, would influence the behavioral actions of ketamine. The efficacy of AAV2 ${ }^{\text {Glun2AshRNA }}$ was confirmed by Cre-dependent GluN2A knockdown in primary neuronal cultures (Supplemental Figure 6, A-D). In male $S s t^{\text {Cret }}$ mice with infusion of AAV2 $2^{\text {Glun2AshRNA }}$ into the $\mathrm{mPFC}$ (Sst ${ }_{\text {Cret/AAV2A }}$ ), there was no significant effect on baseline immobility time in preswim compared to WT-Sst ${ }^{\text {Cre- }}{ }^{\text {AAV2A }}$ control mice (Supplemental Figure 6F). Ketamine administration significantly decreased immobility time in the FST in WT-Sst ${ }^{\mathrm{Cr}-/ A A V-2 A}$ and $S s t^{\mathrm{Cre}+/ \mathrm{AAV}-2 \mathrm{~A}}$ mice (Supplemental Figure 6G). In the NSFT, ketamine administration significantly reduced the latency to feed in WT-Sst $t^{\text {Cre- }}$ AAV2A and $S s t^{\text {Cret/AAV2A }}$ mice (Supplemental Figure 6I). No significant differences were observed in locomotor activity or home cage feeding after ketamine administration (Supplemental Figure 6, H and I).

Genetic deletion of Grin $2 b$ from Sst interneurons. In addition to the virus-mediated approach, we generated Sst interneuronspecific Grin $2 b$ deletion mice by crossing $S s t^{\text {Cret }}$ and Grin $2 b^{f / f l}$ mice (Sst ${ }^{\text {CretGrin2bfl })}$ and littermate controls (Sst $\left.t^{\text {Cre-Grin2bfl}}\right)$. Baseline immobility in the FST and anxiety-related behaviors in the OFT were tested in adult male and female mice (Figure 5A). Similar to virus-mediated knockdown of GluN2B from Sst interneurons in the $\mathrm{mPFC}$, male but not female $S s t^{\mathrm{Cre}+\text { Grinzbfl }}$ mice showed reduced immobility in the FST compared with controls (Figure 5C). There were no differences observed in the OFT (center time or distance traveled) in either sex (Figure 5, D and E). In male $S s t^{{ }^{\text {Cre+Grinzbl }} \text { mice, }}$ there was also a significant antidepressant-like effect (increased time sniffing) in the FUST compared with Sst ${ }^{\text {Cre-Grinzbfl }}$ controls (Figure $5 \mathrm{~F}$ ). In preliminary studies, we found that male and female $S s t^{\text {Cre+Grinzbfl }}$ mice responded similarly to ketamine (Supplemental Figure 7), so we used a mixed cohort of males and females (24 hours after dosing). Ketamine significantly reduced immobility time in the FST in $S s t^{\text {Cre-Grinzbll }}$ controls, but not in $S s t^{\text {CretGrinzbfl }}$ deletion mutants (Figure $5 \mathrm{G}$ ). Ketamine also significantly reduced latency to feed in NSFT in the $S s t^{\text {Cre-Grinzbfl }}$ controls, but not in $S s t^{\text {Cre+Grin2bfl }}$ deletion mice (Figure $5 \mathrm{H}$ ).

To examine the effects of genetic Grin $2 b$ deletion on neuronal activity, postsynaptic currents were recorded from layer $\mathrm{V}$ pyramidal neurons of the MPFC (Figure 5, I and J). Sst ${ }^{\text {Cre+Grinzbfl males }}$ displayed significantly decreased sIPSCs but increased sEPSCs (Figure 5J), similar to in vitro incubation of PFC slices with $1 \mu \mathrm{M}$ ketamine (Figure 1C), whereas Sst ${ }^{\text {Cre+Grinzbfl }}$ females displayed significant decreases of both sIPSCs and sEPSCs (Figure 5K). The increase in sEPSCs in male mice could contribute to the baseline antidepressant-like effects observed in male versus female $S s t^{\text {Cre+Grinzbfl }}$ deletion mutant mice (Figure 5C).

Knockdown of GluN2B in Pvalb interneurons blocks or occludes the antidepressant-like effects of ketamine. To further examine GAB-
Aergic interneuron subtypes in the actions of ketamine and to compare with Sst results, we conducted GluN2B-knockdown studies in Pvalb interneurons. Preliminary studies demonstrated that Pvalb interneurons were responsive to GluN2B-containing NMDARs (blocked by conantokin G) (Supplemental Figure 3) and that Pvalb ${ }^{+}$interneurons in the mPFC expressed GluN2B (Supplemental Figure 2, C and D). Functional knockdown was also shown in Pvalb ${ }_{\text {Cret/AAV }}$ male mice by slice physiology, with decreased NMDAbut not AMPA-induced inward currents (Figure 2J). Using the same design described above, male and female Pvalb ${ }^{\text {Cre+ }}$ or WT-PvalbCrelittermate controls were infused with AAV2 ${ }^{\text {Glun2BshRNA }}$ ( $P$ valb ${ }^{\text {Cret/AAV }}$ or WT-Pvalb ${ }^{\text {Cre-/AAV) }}$ (Figure 6A), resulting in cell-specific recombination with a subset of mCherry only-labeled cells (Figure 6B). In contrast, in WT-Pvalb ${ }^{\mathrm{Cre}-\mathrm{AAV}}$ mice, the labeled neurons were yellow (mCherry + GFP labeled interneuron), demonstrating that there was no recombination. In Pvalb ${ }^{\mathrm{Cre}+\mathrm{AAV}}$ male and female mice, there was no difference in baseline preswim immobility or OFT behavior compared to WT-Pvalb ${ }^{\text {Cre-AAV }}$ controls (Figure 6, C-E). A single dose of ketamine significantly decreased immobility time

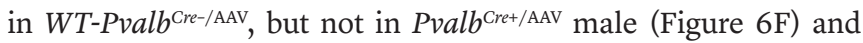
female mice (Figure $6 \mathrm{H}$ ). Interestingly, $P$ valb ${ }^{\text {Cret/AAV }}$ female mice given saline showed significantly reduced baseline immobility in the FST, and there was no further effect of ketamine (Figure $6 \mathrm{H})$; there was a similar trend for decreased immobility in salinetreated Pvalb ${ }^{\text {Cret/AAV }}$ males. In the NSFT, ketamine significantly reduced the latency to feed in male and female WT-Pvalb ${ }^{\text {Cre-/AAV }}$ control mice compared with saline (Figure 6, G and I). In male and female $P$ valb ${ }^{\mathrm{Cre} / \mathrm{AAV}}$ mice, there was no baseline effect in the NSFT, and there were no significant differences after ketamine administration, although there was a trend for a decrease in males (Figure $6, \mathrm{G}$ and I). No differences were observed in home cage feeding (Figure 6, G and I). Next, we measured sIPSCs and sEPSCs in layer $\mathrm{V}$ pyramidal neurons of $\mathrm{mPFC}$ slices from $\mathrm{Pvalb}^{\mathrm{Cre} / \mathrm{AAV}}$ mice (Supplemental Figure 8). In Pvalb ${ }^{\mathrm{Cre}+\mathrm{AAV}}$ males, there was a significant decrease in the frequency of sIPSCs (Supplemental Figure 8, A and B). In contrast to $S s t^{\mathrm{Cre} / \mathrm{AAV}}$ male findings, we observed a small but significant decrease in the frequency of sEPSCs in Pvalb $\mathrm{Cret}^{\mathrm{AAV}}$ males (Supplemental Figure 8, A and B).

\section{Discussion}

The results of the present study demonstrated that GluN2B knockdown in GABAergic interneurons (Gad1, Sst, and Pvalb), but not Camk2a/glutamate neurons, occluded and/or blocked the antidepressant behavioral actions of ketamine (Table 1). The results also provided electrophysiological evidence that a low concentration of ketamine blocked NMDARs on GABA interneurons in the $\mathrm{MPFC}$, resulting in decreased inhibitory input and increased sEPSCs on layer V principle neurons. Viral knockdown or genetic deletion of GluN2B on Sst interneurons produced a similar significant increase (disinhibition) of EPSCs, but only in male mice, which could account for the sex difference in baseline behaviors. Together, these studies provide strong evidence in support of the disinhibition hypothesis that the initial trigger for ketamine is blockade of GluN2B-containing NMDARs on GABAergic interneurons in the $\mathrm{mPFC}$. The $\mathrm{mPFC}$ layer $\mathrm{V}$ principle neurons project to cortical and subcortical brain regions implicated in depression-related behaviors, including the basolateral nucleus of the 
A<smiles>c1ccc(C2C3CC4CC(C3)CC2C4)cc1</smiles>

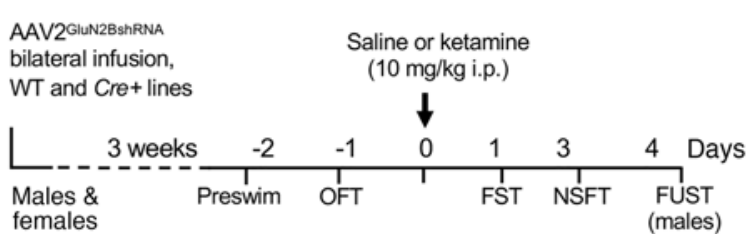

B

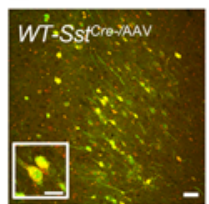

C

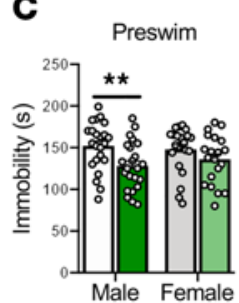

D

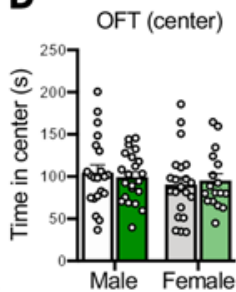

E
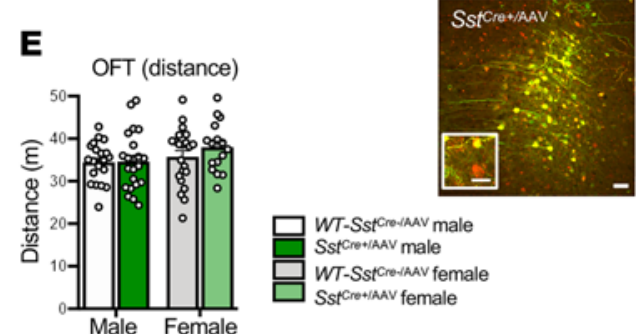

WT-Sstcrovinav male Sstcreotian male WT-Sstrordar female Sstriot/an female

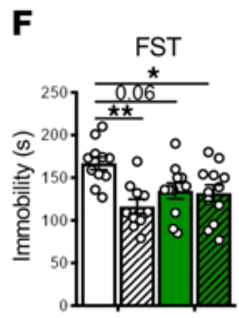

G
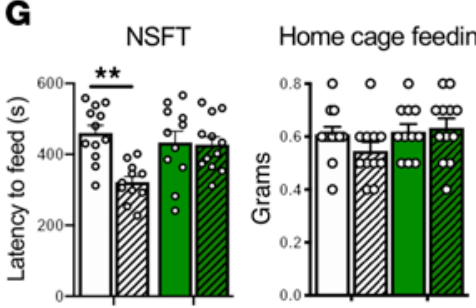

H

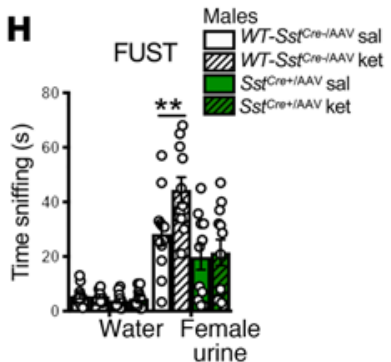

I

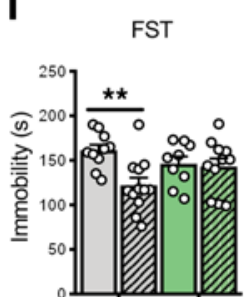

J

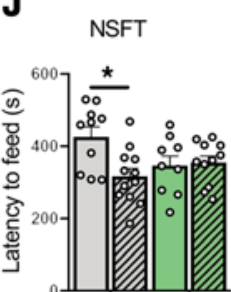

Home cage feeding
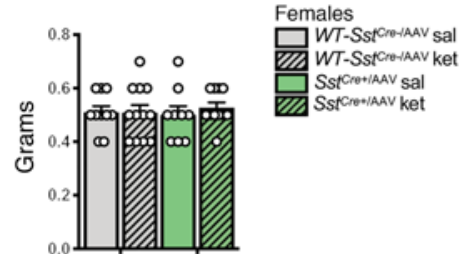
Sstronotians sal SstCrer/AAV sal SstCro/AaV ke

ret
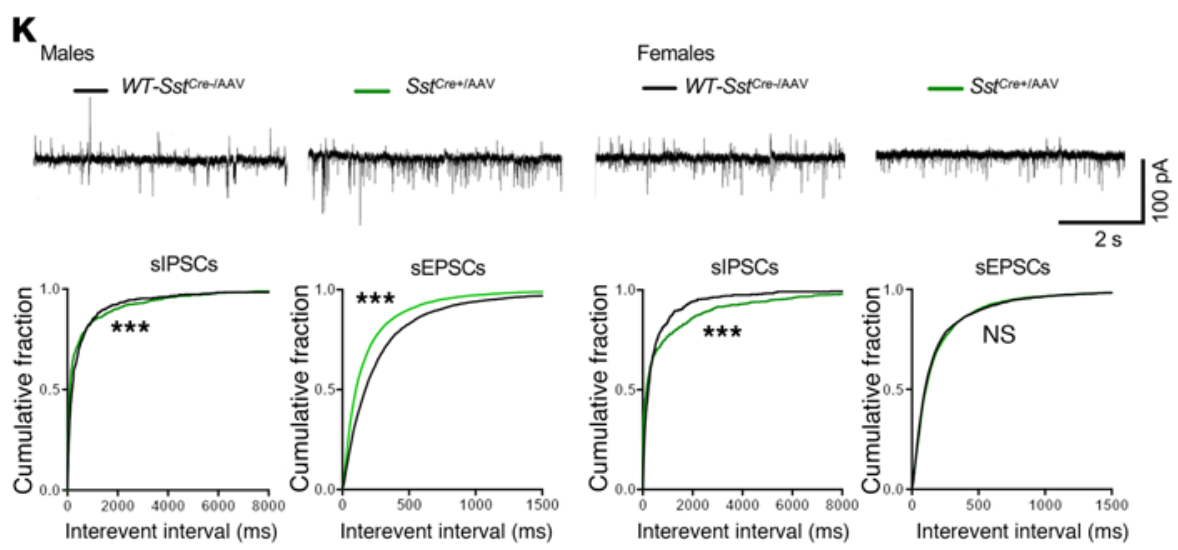

Figure 4. AAV2 $2^{\text {Glun2BshRNA }}$ into the mPFC of Sst ${ }^{\text {Cre+ }}$ mice produces sex differences in baseline behavior and blocks the antidepressant effects of ketamine. (A) Procedure schematic. (B) Representative images of viral expression. Scale bars: $50 \mu \mathrm{m}$ and $20 \mu \mathrm{m}$ (insets). (C) GluN2B knockdown in Sst ${ }^{\text {Cre+/AAV }}$ mice reduced baseline immobility (preswim) in males compared with sex-matched controls ( $n=23$ males, 20-22 females/group, males: $\mathrm{t}_{44}=2.806, P=$ $0.0075)$, but did not affect time spent in center or distance traveled in the OFT ( $\mathbf{D}$ and $\mathbf{E} ; n=$ 21-22 males, 17-22 females/group). (F and I)

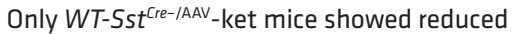
immobility in the FST compared with controls: $n=10-12(\mathbf{F})$ and 9-12 (I) per group (males treatment: $F_{1,40}=9.248, P=0.0041$, genotype times treatment: $\mathrm{F}_{1,40}=7.453, P=0.0094$; females - treatment: $F_{1,38}=6.567, P=0.0145$, genotype times treatment: $F_{1,38}=4.744, P=$ 0.0357). (G and J) Only WT-Sst ${ }^{\text {Cre-/AAV }}$-ket mice showed reduced latency to feed in the NSFT: $n=11-12$ (G) and 9-12 (J) per group (males - treatment: $F_{1,42}=9.171, P=0.0042$, genotype times treatment: $F_{1,42}=7.716, P=$ 0.0081; females - treatment: $F_{1,38}=4.454, P$ $=0.0415$, genotype times treatment: $F_{1,38}=$ 6.176, $P=0.0175$ ). (G and J) No differences were observed in home cage feeding. (H) Only male WT-Sst ${ }^{\text {Cre-/AAV }}$-ket mice showed increased time sniffing female urine in the FUST compared with controls ( $n=11-12$ per group, genotype: $F_{1,84}=102.6, P<0.0001$, treatment: $F_{3,84}=6.199$, $P=0.0007$, genotype times treatment: $F_{3,84}=$ 5.065, $P=0.0028$ ), with no differences in time sniffing water. (K and $\mathbf{L})$ Representative traces of sIPSCs or sEPSCs in layer V pyramidal neurons. (K) Sst ${ }^{\text {Cre+/AAV }}$ males had decreased sIPSCs and increased sEPSCs compared with controls ( $n=25-36$ cells, 8-9 mice) (L) Sst ${ }^{\text {Cre+ }}$ females had decreased sIPSCs, but no differences on sEPSCs compared with controls ( $n=12-19$ cells, 5 mice). Behavioral data are represented as mean \pm SEM. Preswim, OFT: unpaired 2-tailed t test. FST, NSFT: 2-way ANOVA with Tukey's multiple-comparisons test. FUST: 2-way ANOVA with Sidak's multiple-comparisons test. Electrophysiology data are represented as cumulative probability of the interevent interval (IEI). IEIs: Kolmogorov-Smirnov 2-sample test. ${ }^{*} P<0.05$; ${ }^{*} P<0.01$; ${ }^{* *} P<0.001$. NS, nonsignificant. amygdala, which is necessary and sufficient for the rapid and long-lasting antidepressant actions of ketamine $(19,21)$. Because behavioral tests were conducted 24 hours after dosing to avoid the acute side effects of ketamine, we also acknowledge the possibility that there could be adaptations that require GluN2B on GABA interneurons that mediate the actions of ketamine.

An early in vivo electrophysiology study reported that ketamine administration resulted in blockade of GABA interneuron activity in the $\mathrm{mPFC}$, which preceded an increase in glutamate neuronal activity, although the association between these 2 events could only be inferred (10). A recent in vitro electrophysiology study found that ketamine disinhibits CA1 pyramidal neurons in hippocampal slices, in agreement with the disinhibition hypothesis (13). Here, we showed that a low concentration of ketamine (1 $\mu \mathrm{M})$, which approximates brain levels after an antidepressant dose of ketamine (18), decreased sIPSCs but increased sEPSCs of layer $\mathrm{V}$ pyramidal neurons in male and female mice, consistent with the hypothesis that ketamine blocks NMDARs on tonic-firing GABAergic interneurons, decreasing inhibitory inputs onto excitatory pyramidal neurons. An interesting observation from these exper- 
A

\begin{tabular}{|c|c|c|}
\hline 1 & 2 & 4 Day \\
\hline 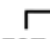 & $T$ & \\
\hline FST & OFT & $\begin{array}{c}\text { FUST } \\
\text { (males only) }\end{array}$ \\
\hline
\end{tabular}

C

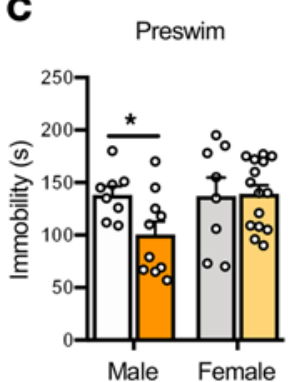

D

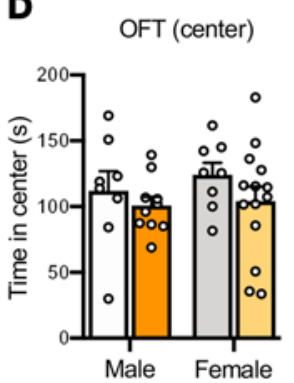

E

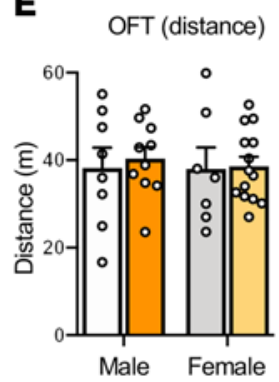

H
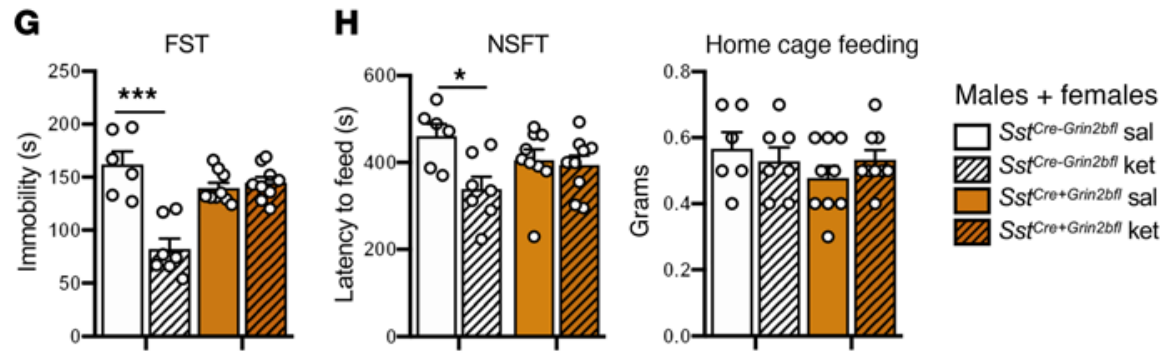

I

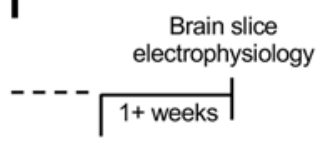

End of behavior

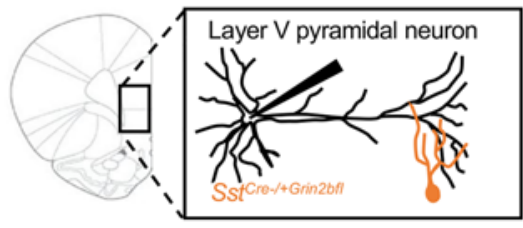

J

Males
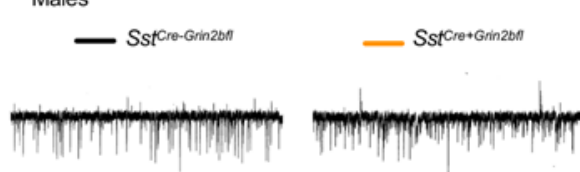

$\mathbf{L}$
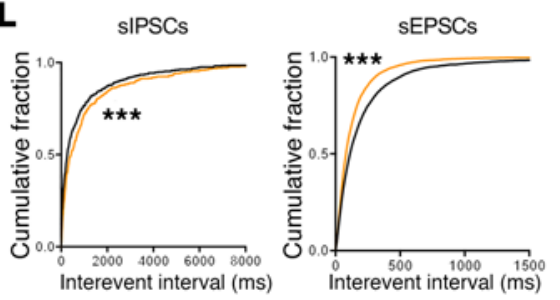

$\mathbf{K}$

Females
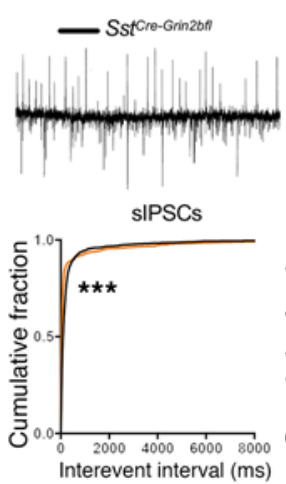

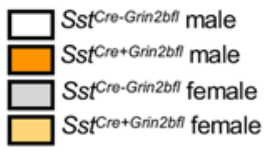

$\mathbf{F}$

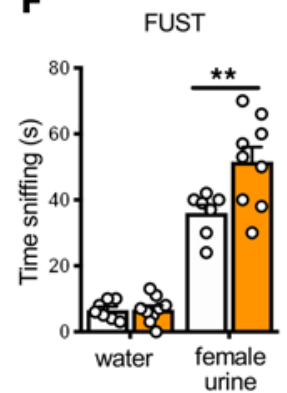

rine

Figure 5. Genetic deletion of Grin2b

from Sst ${ }^{+}$interneurons: behavioral and electrophysiological responses. (A and B) Procedure schematics for baseline (A) and postketamine (B) behaviors. (C) Only Sst ${ }^{\text {Cre+Erinzbfl }}$ male mice had reduced baseline immobility (preswim) when compared with sex-matched controls (Sst ${ }^{\text {Cre-Grinzbfl; }} n=8-10$ males, 8-15 females/group, males: $t_{16}=$ $2.421, P=0.0277)$. ( $\mathbf{D}$ and $\mathbf{E})$ There were no effects in Sst ${ }^{\text {tre+Erinzbfl }}$ mice on baseline time spent in center or distance traveled in the OFT compared with sex-matched controls ( $n=8-10$ males, 8-15 females/group). (F) Male Sst ${ }^{\text {Cre+Crinzbfl }}$ mice showed significantly more time sniffing female urine compared with controls in the FUST ( $n=7-9$ /group, test: $F_{1,28}=163.3, P<0.0001$, genotype: $F_{1,28}$ $=7.244, P=0.0119$, test times genotype: $F_{1,28}=7.069, P=0.0128$ ). (G and $\left.\mathbf{H}\right) \ln \mathrm{a}$ naive group of male and female mice, only control Sst $t^{\text {cre-Crinzbfl-ket mice showed }}$ a significant reduction in (G) time spent immobile ( $n=6-9$ / group, genotype: $F_{1,27}$ $=6.67, P=0.02$, treatment: $F_{1,27}=23.1, P<$ 0.0001 , genotype times treatment: $F_{1,27}=$ 29.5, $P<0.0001)$ and in $(\mathbf{H})$ latency to feed in the novelty-suppressed feeding test (NSFT), when compared with controls ( $n=$ 6-9/group, treatment: $\mathrm{F}_{1,27}=6.54, P=0.02$ treatment times genotype: $F_{1,27}=4.56, P$ $=0.04)$. No significant differences were observed in home cage feeding. (I) Brain slice electrophysiology schematic. (J) Representative traces of sIPSCs and sEPSCs.

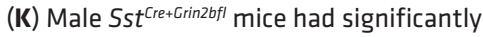
decreased sIPSCs and increased SEPSCs compared with controls ( $n=20-23$ cells, 7-9 mice). (L) Female Sst ${ }^{\text {tret }}$ trinzbfl mice had both significantly decreased sIPSCs and sEPSCs compared with controls ( $n=17-22$ cells, 6-8 mice). Behavioral data are represented as mean \pm SEM. Preswim and OFT: unpaired 2-tailed $t$ test. FUST: 2-way ANOVA with Sidak's multiple-comparisons test. FST and NSFT: 2-way ANOVA with Tukey's multiple-comparisons test. Electrophysiology data are represented as the cumulative probability of the interevent interval (IEI). IEls: Kolmogorov-Smirnov 2-sample test. ${ }^{*} P<0.05$; ${ }^{* *} P<0.01$; ${ }^{* *} P<0.001$. Abbreviations: sal, saline; ket, ketamine.

iments is that female mice had more clustered high-frequency IPSCs compared with males, possibly due to a greater number and/or excitation of burst-firing interneurons in females (29); however, additional studies are needed to address these differences. In contrast, a higher concentration of ketamine $(10 \mu \mathrm{M})$ significantly decreased sIPSCs and sEPSCs in layer $\mathrm{V}$ mPFC neurons of male and female mice, demonstrating the selective effect of the lower $1 \mu \mathrm{M}$ concentration on sIPSCs.

To identify the subunit type and cellular localization of NMDARs underlying the antidepressant action of ketamine, we designed and validated a virus-mediated, Cre recombinase strategy (AAV2 ${ }^{\text {Glun2BshRNA) }}$ to knock down GluN2B or GluN2A on specific populations of neurons in the $\mathrm{MPFC}$, a region that is necessary and sufficient for the actions of ketamine $(2,21)$. The GluN2B subunit was targeted based on evidence that selective GluN2B antagonists produce rapid antidepressant actions in depressed patients and rodent models $(3,16,17)$. The results demonstrated that knockdown of GluN2B on Gad1/GABA interneurons, but not Camk2a/ pyramidal neurons in the $\mathrm{MPFC}$, blocked and/or occluded the antidepressant-like behavioral effects of ketamine (Table 1). GluN2B 
A

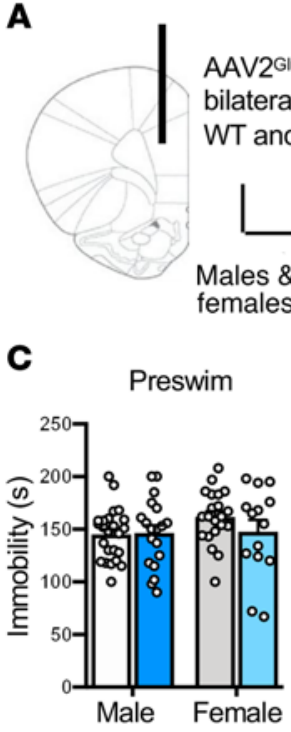

$\mathbf{F}$

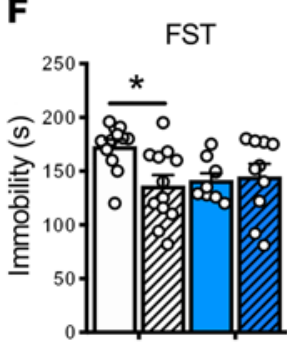

$\mathbf{H}$

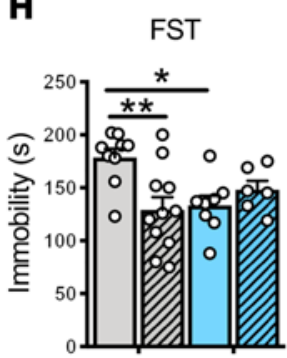

AAV2Glun2BshRNA

bilateral infusion,

WT and Cre+ lines

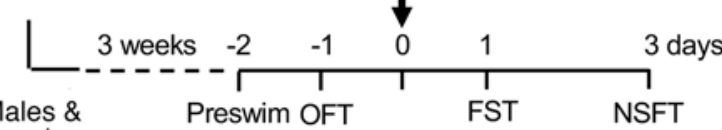

D OFT (center)

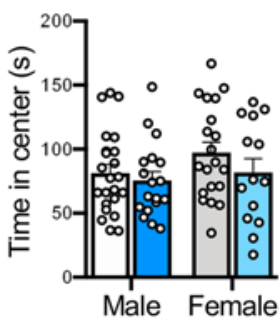

G

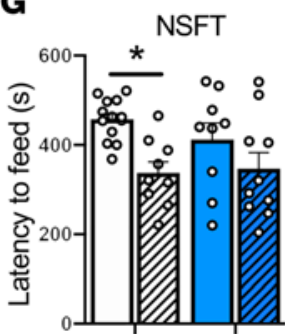

I

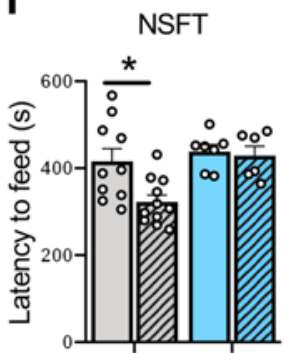

E

B
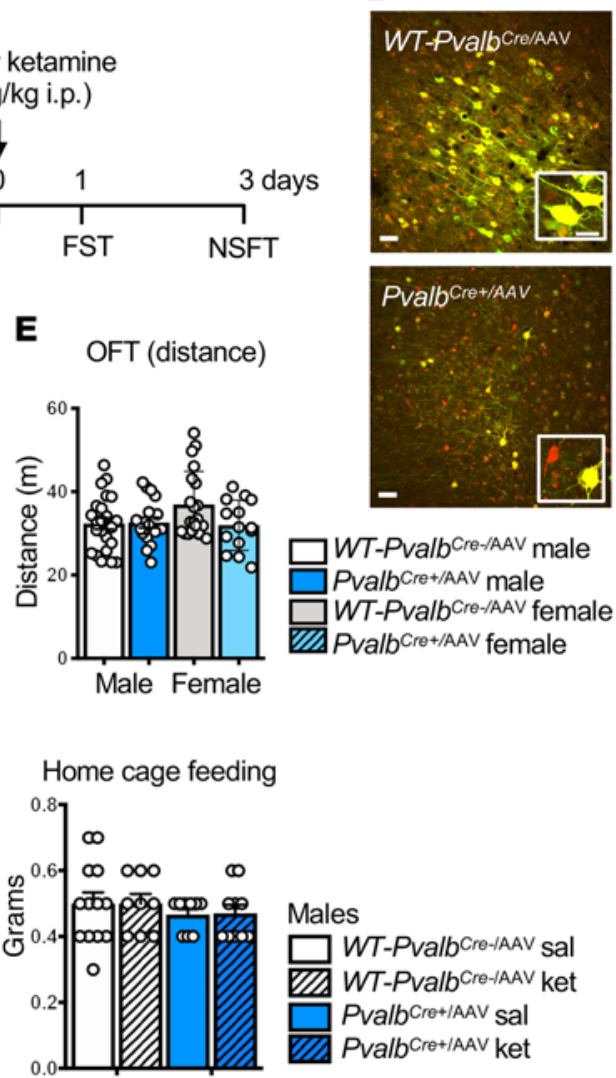

Males

$\square$ WT-PvalbCre-/AAV sal

WT-Pvalb ${ }^{\text {Cre-AAV }}$ ket

PvalbCre+/AAV sal

PvalbCre+/AAV ket

Home cage feeding

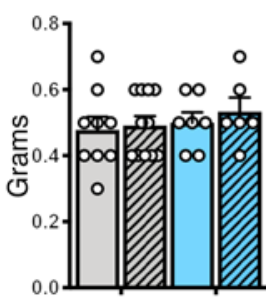

Females

$\square$ WT-PvalbCre-1AAv sal WIA WT-PvalbCre-/AAV ket PvalbCre+/Aav sal $\mathbb{Z}$ PvalbCre+/AAv ket
Figure 6. Infusion of AAV2 $2^{\text {CluN2BshRNA into }}$ the MPFC of Pvalb ${ }^{\text {Cre+ }}$ male and female mice blocks the antidepressant effects of ketamine. (A) Procedure schematic. (B) Representative images of virus-mediated expression and recombination in the $\mathrm{MPFC}$ of WT-Pvalb ${ }^{\text {Cre-/AAV }}$ and Pvalb Cret/AAV $_{\text {mice. }}$ Scale bars: $50 \mu \mathrm{m}$ and $20 \mu \mathrm{m}$ (insets). (C) GluN2B knockdown in Pvalb Cret/Aav $^{\text {mice had }}$ no effect on baseline immobility (preswim; $n=19-24$ males and $14-21$ females per group). ( $\mathbf{D}$ and $\mathbf{E}$ ) GluN2B knockdown in Pvalb ${ }^{\text {Cret/Aav }}$ mice had no effect on baseline time spent in center or distance traveled in the open field test (OFT); however, there was a strong trend toward reduced distance traveled in Pvalb ${ }^{\text {Cret/AAv }}$ females ( $n=18-23$ males and 14-20 females per group). (F and $\mathbf{H}$ ) Only WT-Pvalb Cre-/AAV $^{-}$ ket mice showed significantly reduced immobility in the forced swim test (FST) compared with their saline controls for male $(\mathbf{F})$ and female $(\mathbf{H})$ mice; however, $P$ valb Cre $^{\text {AAAv }}$-sal female mice showed a significant reduction in immobility compared with WT-Pvalb ${ }^{\text {Cre-AAV }}$-sal females: $n=9-12(\mathbf{F})$ and 8-11 $(\mathbf{H})$ per group; males - genotype times treatment: $F_{1,39}=5.1$, $P=0.0291$; females - genotype times treatment: $F_{1,30}=9.094, P=0.0052$. ( $\mathbf{G}$ and I) Only WT-Pvalb Cre-/AAV $_{\text {-ket mice showed }}$ significantly reduced latency to feed in the novelty-suppressed feeding test (NSFT) compared with controls for (C) males and (I) females: $n=9-13$ (G) and 6-11 (I) per group; males - treatment: $F_{1,37}=10.91, P=$ 0.0021; females - genotype: $F_{1,30}=7.782$, $P=0.0091$, treatment: $\mathrm{F}_{1,30}=4.926, P=$ 0.0342 . All data are represented as mean \pm SEM. Preswim and OFT: unpaired 2-tailed $t$ tests performed within sex. FST and NSFT: 2-way ANOVA with Tukey's multiple-comparisons test. ${ }^{*} P<0.05 ;{ }^{* *} P<0.01$. knockdown on Gad1/GABA interneurons also produced baseline antidepressant-like effects on immobility in the preswim test, as well as the FST, a measure of behavioral despair, and occluded any further action of ketamine. To determine whether the occlusion of the ketamine response was due to a floor effect, we tested whether GluN2B knockdown in Gad1 $1^{\text {cret }}$ mice also occluded the response to another antidepressant, DMI, which has a completely different mechanism (i.e., selective inhibition of norepinephrine reuptake). DMI administration produced a significant antidepressant effect in the FST in the Gad1 ${ }^{\text {ret }}$ mice, demonstrating that there was not a floor effect that occluded the response to ketamine. These baseline effects suggest that GluN2B knockdown in Gad1 ${ }^{\text {Cret }}$ mice reduced NMDAR drive of GABA interneurons, similar to ketamine. The reason for the differential baseline effects in the FST and NSFT is unknown, but could result from different cellular or circuit mechanisms that contribute to each behavior. Nevertheless, these results demonstrated a requirement for GluN2B on GABA interneurons in the actions of ketamine.
Our results of GluN2B knockdown in Camk2a/pyramidal neurons differed from a previous study by Hall and colleagues reporting that genetic deletion of Grin $2 b$ using a different excitatory neuron promoter (NEX or Neuro6d) produced baseline antidepressant effects in the FST that occluded the actions of ketamine (30). However, the NEX-Cre ${ }^{+}$Grin $2 b$ deletion mutant mice displayed 3-fold higher levels of locomotor activity than WT controls (31), which could very likely mediate the decreased baseline immobility and occlusion of the ketamine response in the FST (30). We did not observe any changes in locomotor activity in our viral Camk2 $2 a^{\mathrm{Cre} / \mathrm{AAV}}$ or Gad1 $1^{\mathrm{Cre}+/ \mathrm{AAV}}$ knockdown mice, or in any other lines tested. Another study by Hall and colleagues used a viral approach to delete Grin $2 b$ from pyramidal neurons in the mPFC (Grin $2 b^{\sharp / f l}$ mice plus AAV-Camk2a-driven Cre recombinase), reporting decreased baseline immobility time in the FST (32); the influence of ketamine was not examined in this study. The reason for this baseline difference is not clear, but could be due to the complete deletion of Grin $2 b$ in excitatory neurons and targeting 
Table 1. Summary of behavior and electrophysiology findings by sex and genotype

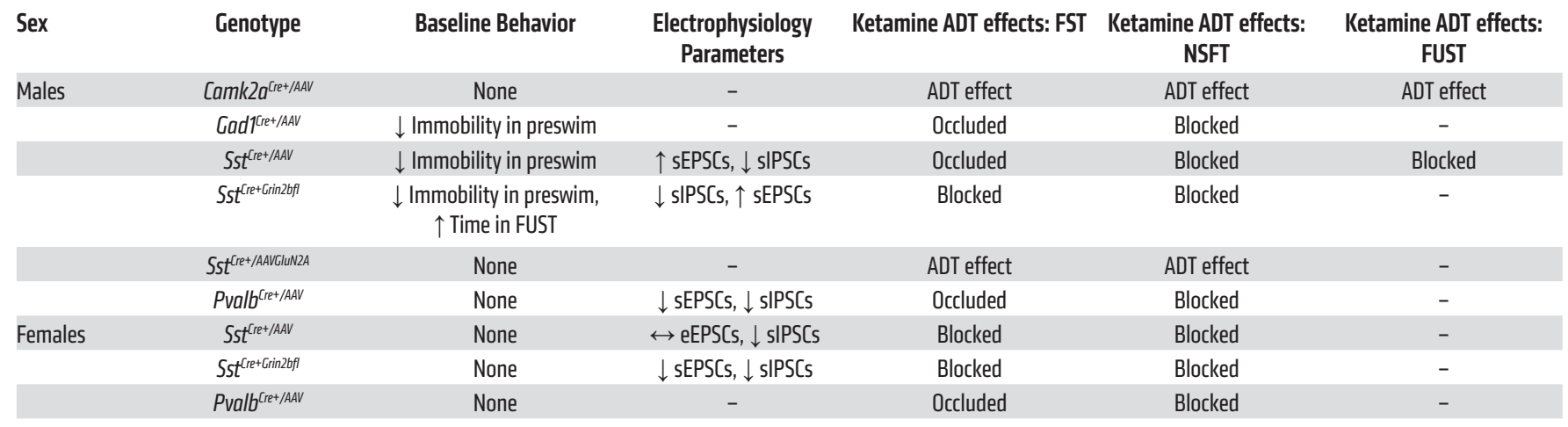

$\uparrow$, increase; $\downarrow$, decrease; $\leftrightarrow$, no change, when compared to controls. ADT, antidepressant; -, no data.

of prelimbic PFC because infralimbic, but not prelimbic, PFC is required for the antidepressant actions of ketamine (21).

We next examined GABA interneuron subtypes, with a focus on Sst interneurons based on evidence from human postmortem studies reporting selective loss of Sst interneurons in the PFC of depressed subjects $(24,25)$ and our previous studies of scopolamine (26). Sst interneurons can be regular spiking, low threshold spiking, and burst firing (15), and blockade of these responses could underlie ketamine-mediated disinhibition. We found that either viral knockdown of GluN2B in the mPFC of $S s t^{\mathrm{Cre}+/ \mathrm{AAV}}$ or constitutive knockout of GluN2B in $S s t^{\text {Cre+Grin2bfl mice occluded }}$ and/or blocked the antidepressant behavioral effects of ketamine in males and females (Table 1). In contrast, knockdown of GluN2A, another prominent subunit in the cerebral cortex, on Sst interneurons did not block the antidepressant effects of ketamine, demonstrating specificity for GluN2B in the actions of ketamine (Table 1). Finally, in viral knockdown and genetic deletion mutant mice, we observed that removal of GluN2B from Sst interneurons produced a significant decrease in preswim immobility in male mice only, indicating a sex-specific role for Sst interneurons in this baseline behavior effect.

Electrophysiological studies of the sex differences showed that GluN2B knockdown in $S s t^{\mathrm{Cre}+/ \mathrm{AAV}}$ or deletion in $S s t^{\mathrm{Cre}+\mathrm{Grin} 2 b f l}$ mice significantly increased sEPSCs in layer $\mathrm{V}$ pyramidal neurons of males, demonstrating an association with antidepressant behavior that was not observed in females. These findings indicate that removal of GluN2B subunits from Sst interneurons resulted in disinhibition of the pyramidal cells, similar to the effects of ketamine. In support of this hypothesis, one study reported that optogenetic inhibition of Sst interneurons increased action-potential firing and burst firing in nearby excitatory neurons in the barrel cortex (33). Why this was not observed in females is unknown, but could be due to different NMDAR and/or AMPAR expression, or synaptic alterations that counter the effects of GluN2B knockdown. Analysis of NMDAR and AMPAR subunit expression revealed no significant sex differences in the relative abundance of Grin1, Grin $2 a$, or Grin $2 b$ in $S s t^{+}$interneurons, but significantly greater expression of Gria2 in females compared with males. Increased Gria2 in female mice could be sufficient to maintain higher tonic firing of $\mathrm{Sst}^{+}$interneurons and inhibitory control of glutamatergic neurons upon knockdown or deletion of Grin2b. Sex differences in NMDAR and/or AMPAR expression on excitatory neurons, which are known to be influenced by estrogen, could also contribute to the observed behavioral effects in males and females $(34,35)$. Female rats have higher baseline PFC GluN1 expression and increased stress-induced expression of GluN2B compared with males (36). Postmortem studies have reported that female depressed subjects had increased expression of several glutamate receptor subtypes in the dorsolateral PFC, and that female depressed suicide subjects had significantly increased expression of Grin $2 b$ (37). Only one study to date has analyzed sex differences in ketamine response and found no differences in efficacy or tolerability $(38,39)$. These studies highlight the need for further studies of sex differences in the behavioral, neural, molecular, and genetic phenotypes of major depressive disorder to inform the development of more effective treatment options (40).

Pvalb interneurons are noted for their fast-spiking properties at rest, and could also be involved in ketamine-mediated disinhibition (15). We found that viral knockdown of GluN2B in the mPFC of Pvalb ${ }^{\text {Cret/AAv }}$ male and female mice blocked the antidepressant behavioral effects of ketamine (Table 1). This differs from our previous study of scopolamine, in which we found that knockdown of M1-AChR on Sst but not Pvalb interneurons blocked the antidepressant effects of scopolamine (26), raising the possibility that the disinhibition resulting from the actions of ketamine on both Sst and Pvalb interneurons contributes to its greater therapeutic efficacy relative to scopolamine (41). Indeed, animals that have been subjected to inescapable footshock have reduced excitatory drive onto prefrontal Pvalb interneurons, and chemogenetic activation of these interneurons can lead to susceptibility in resilient mice, suggesting that Pvalb interneurons are also important in regulating depression-like behavioral output (42). Saline-treated Pvalb ${ }^{\text {Cret/AAV }}$ mice displayed decreased immobility, which could result from blockade of increases that are observed with repeated swim exposure that is observed in rodents, suggesting a role for GluN2B subunits on mPFC Pvalb interneurons in memory or stress resiliency processes. For example, blocking synaptic transmission in mPFC Pvalb interneurons impairs spatial working memory and reversal learning, but has no effect on anxiety as measured by the OFT (43). A previous study reported that constitutive global genetic 
deletion of Grin1 from Pvalb ${ }^{\text {Cret }}$ mice did not block the effects of ketamine (44). The differences in the scope and developmental targeting of Grin1 deletion in Pvalb interneurons could underlie the different behavioral outcomes in these studies.

The results of the current study provided direct evidence that the initial cellular trigger for the antidepressant actions of ketamine was blockade of NMDARs on GABAergic interneurons, not excitatory neurons, leading to disinhibition and a burst of glutamate that drives synaptic and behavioral responses. Ketamine blockade of GABA activity appeared to be in opposition to evidence of a reduction of GABA, particularly Sst interneurons, in depression $(24,25)$. Our results also appeared to contradict rodent studies reporting that increased Sst interneuron function produced antidepressant behavioral responses (45). However, ketamine blockade of NMDARs and the resulting glutamate burst were transient, returning to baseline levels by 80 minutes after dosing (5), but led to long-lasting synaptic and antidepressant behavioral actions $(2,22,46,47)$. In addition, recent work reported that a single dose of ketamine also led to increased GABA function in the mPFC 24 hours after dosing, evidence of further adaptations to the initial glutamate burst that would contribute to correction of GABA, as well as glutamate deficits (48). This could also explain the apparent contradictory effects reported in another rodent study, which found that repeated ketamine administration increased Pvalb interneuron activity in juvenile stressed mice (49), but this likely represents an adaptation to repeated induction of the glutamate burst. Thus, the impact of transient versus sustained effects is critical when interpreting these experiments.

In conclusion, conventional monoaminergic antidepressant treatments take weeks to months to produce a therapeutic response and are ineffective for treatment-resistant depression. The novel, rapid-acting antidepressant actions of ketamine are effective even for patients considered treatment resistant, but the abuse potential of ketamine remains a limitation. Dissection of the cellular and molecular mechanisms underlying ketamine's antidepressant effects could lead to the development of more targeted and safer treatments for depression. The results of the current study reveal an important cellular mechanism by which ketamine produces rapid antidepressant actions, indicating that activity-related neurotransmitter receptors and channels on Sst and Pvalb interneurons in the $\mathrm{MPFC}$ are promising targets for the development of novel rapid-acting antidepressants.

\section{Methods}

Animals. For electrophysiology experiments looking at the effects of ketamine, WT C57BL/6 male and female mice were used (Jackson Laboratories). For electrophysiology experiments looking at the effects of ketamine or conantokin G on NMDA-induced inward currents, WT C57BL/6, Sst-tdTomato, or Pvalb-tdTomato male mice were used. To obtain Sst- and Pvalb-tdTomato mice, Sst-Cre and Pvalb-Cre mice were bred with Ai9(RCL-tdT) mice (catalog 007909), all from Jackson Laboratories. For FACS and RNA-Seq, Sst-tdTomato male and female mice were used. For viral studies, male and female transgenic mice and WT C57BL/6 littermates were obtained from in-house breeders. Gad1-Cre mice were originally obtained from Marina Picciotto (Yale School of Medicine) (50), and Camk2a-Cre mice were obtained from Günter Schütz (German Cancer Research Center, Heidelberg, Germany) (51). Pvalb-Cre (catalog 008069) and Sst-Cre (catalog 013044) mice were obtained from Jackson Laboratories. Grin $2 B^{f / f l} / S s t-C r e^{+}$mice were produced by crossing Grin $2 b$ floxed mice (Eric Delpire, Vanderbilt University, Nashville, Tennessee, USA) (52) with Sst-Cre heterozygous mice to produce Grin2 $B^{f / f l} / S s t-C r e^{+}$and Grin $2 B^{f / f l} / S s t-C r e^{-}$offspring. For lines that required additional breeding, at least 6 backcrosses were performed. All studies were performed with adult mice. Pregnant female rats were used as a source of embryonic tissue for primary neuronal cultures (Jackson Laboratories). Animals were housed in standard ventilated rack under a 12-hour light/12-hour dark cycle with ad libitum access to water and rodent chow. All experiments were performed during the 12-hour light cycle. Animal use and procedures were in accordance with the NIH guidelines and approved by the Yale University Animal Care and Use Committees.

Brain slice electrophysiology. Brain slices containing the $\mathrm{mPFC}$ were prepared from male and female mice (8 to 16 weeks old). Brains were placed in artificial cerebrospinal fluid (ACSF) ( $\mathrm{pH}$ 7.35-7.38) equilibrated with $95 \% \mathrm{O}_{2} / 5 \% \mathrm{CO}_{2}$. Coronal slices of $300-\mu \mathrm{m}$ thickness containing the $\mathrm{mPFC}$ were transferred to the fixed stage of an Olympus BX50WI scope for whole-cell recording. The chamber was continuously perfused with normal ACSF at a rate of 2 to $3 \mathrm{~mL} /$ minute and its temperature maintained at $33^{\circ} \mathrm{C} \pm 0.5^{\circ} \mathrm{C}$. Patch pipettes $(3-5$ $\mathrm{M} \Omega$ ) were pulled from glass tubing with a Flaming/Brown horizontal puller. The pipette solution contained the following: $115 \mathrm{mM} \mathrm{K}$ gluconate, $5 \mathrm{mM} \mathrm{KCl}, 2 \mathrm{mM} \mathrm{MgCl}_{2}, 2 \mathrm{mM} \mathrm{Mg}$-ATP, $2 \mathrm{mM} \mathrm{Na}_{2} \mathrm{ATP}, 10 \mathrm{mM}$ $\mathrm{Na}_{2}$-phosphocreatine, $0.4 \mathrm{mM} \mathrm{Na}_{2} \mathrm{GTP}$, and $10 \mathrm{mM}$ Hepes, $\mathrm{pH}$ 7.33. Neurons were visualized by videomicroscopy using a microscope (40× IR lens) with infrared differential interference contrast (IR/DIC). Whole-cell recordings were made with an Axoclamp-2B amplifier. Postsynaptic currents were studied in the continuous single-electrode voltage-clamp mode (3000 Hz low-pass filter). sIPSCs and sEPSCs were simultaneously recorded (53-55) at a holding potential of -65 $\mathrm{mV}$. Because the reversal potential of $\mathrm{Cl}^{-}$was $-72 \mathrm{mV}$ (calculated from pipette solution), at $-65 \mathrm{mV}$ membrane potential, sIPSCs and sEPSCs were distinguished by their outward and inward polarity, respectively. Synaptic events were analyzed using Mini Analysis Software (Synaptosoft). We tested $10 \mu \mathrm{M}$ NMDA- and $5 \mu \mathrm{M}$ AMPA-induced inward currents with no added magnesium in the ACSF.

Immunohistology. After 3 weeks to allow for viral expression, brains were collected from mice infused with AAV2GluN2BshRNA after transcardiac perfusion with sterile PBS and $4 \%$ paraformaldehyde (PFA). Brains were postfixed in 4\% PFA for 24 hours and incubated in $30 \%$ sucrose for an additional 24 hours. Fixed brains were frozen and sectioned at $30 \mu \mathrm{m}$ using a Microm HM550 cryostat. To examine the colocalization of GluN2B with different cell markers, brain sections were washed and incubated with blocking buffer (PBS, $0.3 \%$ Triton $\mathrm{X}-100,4 \%$ normal goat serum; 1 hour at room temperature), followed by overnight incubation with primary antibodies at $4^{\circ} \mathrm{C}$ : anti-Camk2a (Enzo Life Sciences, catalog KAM-CA002-D, 1:1000, rat), anti-GAD67 (Sigma-Aldrich, catalog G5419, 1:1000, mouse), and anti-GluN2B (EMD Millipore, catalog AB1557, 1:500, rabbit). Brain sections from Sst-tdTomato and Pvalb-tdTomato reporter mice were used for immunolabeling of GluN2B on $S s t^{+}$and Pvalb interneurons (anti-GluN2B, Alomone Labs, catalog AGC-003, 1:200, rabbit). To match the style of Camk2a and GAD67 images, Sst ${ }^{+}$and 
$\mathrm{Pvalb}^{+}$stains were flipped to green, and GluN2B stains were flipped to red. Sections were then washed and incubated overnight at $4^{\circ} \mathrm{C}$ or 2 hours at room temperature with fluorescently conjugated secondary antibodies (Alexa Fluor, Thermo Fisher Scientific, 1:1000 dilution). Immunofluorescence was visualized using a confocal laser scanning microscope (Olympus FV1000).

shRNA and virus preparation. For knockdown of GluN2B or GluN2A in specific neuronal cell types, an shRNA sequence was designed targeting the GluN2B or GluN2A subunit (56). GluN2B shRNA (5'-TgtaccaacaggtctcaccttaaacTTCAAGAGAgtttaaggtgagacetgttggtacTTTTTTC-3') or GluN2A shRNA (5'-TtgacagaacgcgaacttcgaaatcTTCAAGAGAgatttcgaagttcgcgttctgtcaTTTTTTC-3'; Integrated DNA Technologies) was ligated into a plasmid (pGluN2BshRNA or pGluN2AshRNA) designed to restrict shRNA expression to cells that express Cre recombinase (57). The TATA-loxP-flanked CMV-EGFP cassette was subcloned from pSico (from Tyler Jacks, Massachusetts Institute of Technology, Boston, Massachusetts, USA; Addgene plasmid 11578) into pAAV-mCherry (Virovek) to generate the sMACKdown backbone. This plasmid allows ubiquitous mCherry expression and conditional EGFP expression driven by floxed CMV cassette that prevents shRNA expression with U6 promoter disruption.

Primary cortical cultures. Pregnant female rats were euthanized and cortices from E18 embryos were dissected. After incubation in trypsin-EDTA $(0.25 \%$, Gibco) for 10 minutes, cortices were dissociated and neurons were plated at 1 million cells per well in 6-well poly-D-lysine-coated plates in DMEM (Gibco)/10\% FBS. Medium was changed the following day to a serum-free medium containing neurobasal and B27 (Gibco), which was changed every 4 days. Cells were maintained at $37^{\circ} \mathrm{C}, 5 \% \mathrm{CO}_{2}$, and $95 \%$ humidity. On day in vitro (DIV) $5,0.4 \mu \mathrm{L} \mathrm{AAV2} 2^{\text {Glun2BshRNA }}$ or AAV2 ${ }^{\text {GluN2AshRNA }}$, AAV1-Cre, or both viruses in combination were added to the wells. After 21 days (DIV26) of viral infection and shRNA expression, cells were collected for Western blot analysis.

Western blot analysis. Levels of GluN2B or GluN2A from cell lysates were analyzed by Western blot analysis. Cells were collected into RIPA lysis buffer containing $50 \mathrm{mM}$ Tris- $\mathrm{HCl}$ (pH 7.5), $150 \mathrm{mM}$ $\mathrm{NaCl}, 1 \%$ Triton $\mathrm{X}-100,0.1 \% \mathrm{SDS}, 1 \mathrm{mM} \mathrm{NaVO}_{3}, 5 \mathrm{mM} \mathrm{NaF}$, and $1 \times$ protease inhibitor cocktail. Homogenates were then centrifuged at $16,200 \mathrm{~g}$ for 10 minutes to remove cell debris. The supernatant was collected and total protein concentrations measured using a protein assay kit (Pierce BCA Protein Assay Kit, Thermo Fisher Scientific). Twenty micrograms of protein was electrophoretically separated in an SDS-PAGE gel (7.5\% Tris- $\mathrm{HCl})$ and transferred to $2-\mu \mathrm{m}$ polyvinylidene fluoride or PVDF membranes. Membranes were blocked with 5\% BSA in PBS plus 0.05\% Tween 20 (PBS-T) and then incubated with GluN2B antibody (Ab) (Cell Signaling Technology, catalog 4307, 1:1000; rabbit) or GluN2A Ab (BD biosciences, catalog $612286,1: 1000$, mouse) overnight. Membranes were then washed in PBS-T (3 times for 10 minutes each) and incubated in peroxidase-labeled anti-rabbit (Vector Laboratories, catalog PI-1000, 1:10,000) or anti-mouse secondary (Cell Signaling Technology, cat$\operatorname{alog} 7076,1: 10,000)$ at room temperature for 1 hour. Protein bands were analyzed using ECL. After detection of GluN2B or GluN2A, membranes were incubated in stripping buffer (Thermo Fisher Scientific), blocked in 5\% BSA in PBS-T for 1 hour, and then reprobed for $\beta$-actin (Cell Signaling Technology, catalog 4970, 1:1000) overnight. Densitometry was used to quantify protein bands (Image Lab,
Bio-Rad) and GluN2B or GluN2A were normalized to their respective $\beta$-actin level (loading control).

Surgery and cortical infusion. Mice were anesthetized with a ketamine/xylazine (100/10 mg/kg) cocktail. Bilateral viral infusions into the $\mathrm{mPFC}(0.5 \mu \mathrm{L}$ per side; $0.1 \mu \mathrm{L} /$ minute $)$ were performed with coordinates (from bregma) as follows: $+2.0 \mathrm{~mm}$ anterior-posterior, \pm 0.4 $\mathrm{mm}$ medial-lateral, and $-2.8 \mathrm{~mm}$ dorsal-ventral (58). Incisions were closed with sutures, and an antibiotic was applied to prevent infection. Mice received i.p. injection of carprofen $(5 \mathrm{mg} / \mathrm{kg})$ immediately after surgery and daily for the next 2 days.

Drug administration. For ketamine experiments, mice received 1 injection of vehicle (0.9\% saline) or ketamine ( $10 \mathrm{mg} / \mathrm{kg}$, i.p.) 24 hours before behavioral testing. For DMI experiments, mice received 1 injection of vehicle (0.9\% saline) or DMI ( $20 \mathrm{mg} / \mathrm{kg}$, i.p.) 30 minutes before behavioral testing.

OFT. The OFT was conducted in a Plexiglas test apparatus $(40 \times$ $40 \times 25 \mathrm{~cm}$ ) and recorded for 10 minutes. Activity in the OFT was analyzed using an automated system (ANY-maze, Stoelting).

FST. FST, referred to as preswim if administered prior to drug treatment, was conducted as previously described (2). Mice were placed for 6 minutes in a clear cylinder filled with water $\left(24^{\circ} \mathrm{C} \pm 1^{\circ} \mathrm{C}, 18\right.$ $\mathrm{cm}$ depth). Sessions were video recorded and scored for total immobility time by a blinded experimenter. Time immobile during the 2- to 6-minute block was reported.

NSFT. NSFT was conducted as previously described (59). Mice were food deprived for 18 hours and placed in a dimly lit box $(40 \times$ $40 \times 25 \mathrm{~cm}$ ) with food in the center. The latency to feed was recorded. Home cage food intake over a 15 -minute period was measured as a feeding control.

RNA preparation and RNA-Seq. In brief, the MPFC was dissected from Sst-tdTomato reporter mice and incubated in DMEM (GIBCO) $/ 0.25 \%$ trypsin (GIBCO) for 30 minutes at $37^{\circ} \mathrm{C}$. The tissue pieces were then homogenized using a $20 \% \mathrm{FBS} / \mathrm{HBSS} / 0.2 \%$ BSA solution, centrifuged (1200 $g$ for 6 minutes) to collect the cells and resuspended and filtered using HBSS/0.2\% BSA solution. Fluorescent cells were sorted and collected into $100 \mu \mathrm{L}$ of lysis buffer (Norgen kit) using FACS (FACSCalibur, Becton Dickinson). RNA was extracted using Arcturus PicoPure RNA isolation kit (Applied Biosystems). Using the Pico RiboGone-Mammalian (TakaraBio), 100 ng of total mRNA was processed before cDNA synthesis. RNA-Seq was performed on an Illumina HiSeq 2500 using 75-bp, paired-end sequencing. FASTQ files were trimmed and assessed for quality control before being annotated with the mouse genome (mm9) using TopHat241. The assembled transcripts were used to estimate transcript abundance and fragments per kilobase of transcript per million mapped reads (FPKM) values using cuffdiff42 and $\mathrm{R}$ (The Comprehensive R Archive Network. https://www.cran.r-project.org). RNASeq data were deposited to NCBI Gene Expression Omnibus data set repository (GEO accession GSE138670).

FUST. This test was conducted according to previously published procedures (27). Male mice were habituated for 60 minutes to a sterile cotton-tipped applicator placed in their home cage. For the test, mice were first exposed to a new cotton tip dipped in sterile water; 45 minutes later, mice were then exposed to another cotton tip infused with $45 \mu \mathrm{L}$ fresh urine collected from adult females of the same strain. Estrous cycle was not measured because prior studies have reported that females in all states of estrus elicit higher investigation from 
males than ovariectomized females (60). Behavior was video recorded for 5 minutes per test and total time spent sniffing the cotton tip was determined. Time spent biting the cotton tip was excluded from the recording time.

Locomotor activity. Animals were placed in a clean testing cage (30 $\times 19 \times 13 \mathrm{~cm}$ ) for 30 minutes, during which the number of beam breaks was measured using the Med-PC software (Med Associates).

Statistics. Data were subjected to statistical analyses with GraphPad Prism 8. The results are expressed as the mean \pm SEM. Statistical significance was determined by a 1-way analysis of variance (ANOVA) or a 2-way ANOVA, followed by the Tukey's or Sidak's (FUST) multiplecomparisons test for comparing the treated group with the control group and multigroup comparisons, as indicated in the figure legends. Statistical differences between any 2 groups were determined using either the Student's 2-tailed $t$ test or Kolmogorov-Smirnov 2-sample test. Across all analyses, differences were considered significant for $P$ values less than 0.05. Any value greater than 2 times the standard deviation of the mean was considered an outlier and removed from further analysis of the test at hand. The specific test used for each figure is mentioned in the figure legend. Each experiment was conducted a minimum of 2 times.

Study approval. Animal use and procedures were in accordance with NIH guidelines and approved by the Yale University Animal Care and Use Committees.

\section{Author contributions}

DMG, SP, ESW, and RSD designed the study. DMG, SP, RJL, MW, MJG, and CHD conducted experiments, analyzed data, and interpreted the results. XYL helped with breeding, genotyping, and the development of the $S s t^{\text {Cre+Grinzbll }}$ mouse line. SRT and MP contributed essential reagents and experimental advice for cell type-selective knockdown experiments. ED provided the Grin $2 b^{f l / f l}$ line and helped with the design of the Grin $2 b^{f l / f l}$ lines used in the study. DMG and RSD wrote the manuscript. DMG was designated as the first co-first author based on primary contributions. All authors reviewed and approved the final manuscript.

\section{Acknowledgments}

This research was supported by National Institute of Mental Health grants MH093897 and MH105910 (to RSD) and the State of Connecticut. SRT and MRP were supported by DA14241 and MH077681. The Glun $2 b^{f / f l}$ mouse was generated by the GeneTargeted Mouse Core of the INIA-stress consortium (grant U01 AA013514 to ED).

Address correspondence to: Ronald Duman, Psychiatry, 34 Park Street, New Haven, Connecticut, 06509, USA; Phone: 203.974.7726; Email: ronald.duman@yale.edu
1. Berman RM, et al. Antidepressant effects of ketamine in depressed patients. Biol Psychiatry. 2000;47(4):351-354.

2. Li N, et al. mTOR-dependent synapse formation underlies the rapid antidepressant effects of NMDA antagonists. Science. 2010;329(5994):959-964.

3. Li N, et al. Glutamate N-methyl-D-aspartate receptor antagonists rapidly reverse behavioral and synaptic deficits caused by chronic stress exposure. Biol Psychiatry. 2011;69(8):754-761.

4. Autry AE, et al. NMDA receptor blockade at rest triggers rapid behavioural antidepressant responses. Nature. 2011;475(7354):91-95.

5. Moghaddam B, Adams B, Verma A, Daly D. Activation of glutamatergic neurotransmission by ketamine: a novel step in the pathway from NMDA receptor blockade to dopaminergic and cognitive disruptions associated with the prefrontal cortex. J Neurosci. 1997;17(8):2921-2927.

6. Gerhard DM, Wohleb ES, Duman RS. Emerging treatment mechanisms for depression: focus on glutamate and synaptic plasticity. Drug Discov Today. 2016;21(3):454-464.

7. Miller OH, Moran JT, Hall BJ. Two cellular hypotheses explaining the initiation of ketamine's antidepressant actions: Direct inhibition and disinhibition. Neuropharmacology. 2016;100:17-26.

8. Constantinidis C, Goldman-Rakic PS. Correlated discharges among putative pyramidal neurons and interneurons in the primate prefrontal cortex. J Neurophysiol. 2002;88(6):3487-3497.

9. Rao SG, Williams GV, Goldman-Rakic PS. Isodirectional tuning of adjacent interneurons and pyramidal cells during working memory: evidence for microcolumnar organization in PFC.
J Neurophysiol. 1999;81(4):1903-1916.

10. Homayoun H, Moghaddam B. NMDA receptor hypofunction produces opposite effects on prefrontal cortex interneurons and pyramidal neurons. J Neurosci. 2007;27(43):11496-11500.

11. Kawaguchi Y. Distinct firing patterns of neuronal subtypes in cortical synchronized activities. J Neurosci. 2001;21(18):7261-7272.

12. Povysheva NV, et al. Properties of excitatory synaptic responses in fast-spiking interneurons and pyramidal cells from monkey and rat prefrontal cortex. Cereb Cortex. 2006;16(4):541-552.

13. Widman AJ, McMahon LL. Disinhibition of CA1 pyramidal cells by low-dose ketamine and other antagonists with rapid antidepressant efficacy. Proc Natl Acad Sci U S A . 2018;115(13):E3007-E3016.

14. DeFelipe J, et al. New insights into the classification and nomenclature of cortical GABAergic interneurons. Nat Rev Neurosci. 2013;14(3):202-216.

15. Tremblay R, Lee S, Rudy B. GABAergic interneurons in the neocortex: from cellular properties to circuits. Neuron. 2016;91(2):260-292.

16. Maeng $\mathrm{S}$, et al. Cellular mechanisms underlying the antidepressant effects of ketamine: role of $\alpha$-amino-3-hydroxy-5-methylisoxazole-4-propionic acid receptors. Biol Psychiatry. 2008;63(4):349-352.

17. Preskorn SH, Baker B, Kolluri S, Menniti FS, Krams M, Landen JW. An innovative design to establish proof of concept of the antidepressant effects of the NR2B subunit selective N-methyl-D-aspartate antagonist, CP-101,606, in patients with treatment-refractory major depressive disorder. JClin Psychopharmacol. 2008;28(6):631-637.

18. Hartvig P, et al. Central nervous system effects of subdissociative doses of (S)-ketamine are related to plasma and brain concentrations measured with positron emission tomography in healthy volunteers. Clin Pharmacol Ther. 1995;58(2):165-173.

19. Hare BD, Shinohara R, Liu RJ, Pothula S, DiLeone RJ, Duman RS. Optogenetic stimulation of medial prefrontal cortex Drd1 neurons produces rapid and long-lasting antidepressant effects. Nat Commun. 2019;10(1):223.

20. Halliwell RF, Peters JA, Lambert JJ. The mechanism of action and pharmacological specificity of the anticonvulsant NMDA antagonist MK-801: a voltage clamp study on neuronal cells in culture. Br J Pharmacol. 1989;96(2):480-494.

21. Fuchikami M, et al. Optogenetic stimulation of infralimbic PFC reproduces ketamine's rapid and sustained antidepressant actions. Proc Natl Acad Sci US A. 2015;112(26):8106-8111.

22. Duman RS, Aghajanian GK, Sanacora G, Krystal JH. Synaptic plasticity and depression: new insights from stress and rapid-acting antidepressants. Nat Med. 2016;22(3):238-249.

23. Markram H, Toledo-Rodriguez M, Wang Y, Gupta A, Silberberg G, Wu C. Interneurons of the neocortical inhibitory system. Nat Rev Neurosci. 2004;5(10):793-807.

24. Seney ML, Tripp A, McCune S, Lewis DA, Sibille E. Laminar and cellular analyses of reduced somatostatin gene expression in the subgenual anterior cingulate cortex in major depression. Neurobiol Dis. 2015;73:213-219.

25. Lin LC, Sibille E. Somatostatin, neuronal vulnerability and behavioral emotionality. Mol Psychiatry. 2015;20(3):377-387.

26. Wohleb ES, et al. GABA interneurons mediate the rapid antidepressant-like effects of scopolamine. JClin Invest. 2016;126(7):2482-2494.

27. Malkesman $\mathrm{O}$, et al. The female urine sniffing 
test: a novel approach for assessing rewardseeking behavior in rodents. Biol Psychiatry. 2010;67(9):864-871.

28. Tasic B, et al. Adult mouse cortical cell taxonomy revealed by single cell transcriptomics. Nat Neurosci. 2016;19(2):335-346.

29. Wang Y, et al. Anatomical, physiological and molecular properties of Martinotti cells in the somatosensory cortex of the juvenile rat. J Physiol (Lond). 2004;561(pt 1):65-90.

30. Miller OH, et al. GluN2B-containing NMDA receptors regulate depression-like behavior and are critical for the rapid antidepressant actions of ketamine. Elife. 2014;3:e03581.

31. Wang CC, et al. A critical role for GluN2Bcontaining NMDA receptors in cortical development and function. Neuron. 2011;72(5):789-805.

32. Miller OH, Bruns A, Ben Ammar I, Mueggler T, Hall BJ. Synaptic regulation of a thalamocortical circuit controls depression-related behavior. Cell Rep. 2017;20(8):1867-1880.

33. Gentet LJ, Kremer Y, Taniguchi H, Huang ZJ, Staiger JF, Petersen CC. Unique functional properties of somatostatin-expressing GABAergic neurons in mouse barrel cortex. Nat Neurosci. 2012;15(4):607-612.

34. Smith CC, Vedder LC, McMahon LL. Estradiol and the relationship between dendritic spines, NR2B containing NMDA receptors, and the magnitude of long-term potentiation at hippocampal CA3-CA1 synapses. Psychoneuroendocrinology. 2009;34(suppl 1):S130-S142.

35. Galvin C, Ninan I. Regulation of the mouse medial prefrontal cortical synapses by endogenous estradiol. Neuropsychopharmacology. 2014;39(9):2086-2094.

36. Wang Y, et al. Prenatal chronic mild stress induces depression-like behavior and sex-specific changes in regional glutamate receptor expression patterns in adult rats. Neuroscience. 2015;301:363-374

37. Gray AL, et al. Sex differences in glutamate receptor gene expression in major depression and suicide. Mol Psychiatry. 2015;20(9):1057-1068.

38. Freeman MP, et al. Sex differences in response to ketamine as a rapidly acting intervention for treatment resistant depression. JPsychiatr Res.
2019;110:166-171.

39. Gerhard DM, Duman RS. Rapid-acting antidepressants: mechanistic insights and future directions. Curr Behav Neurosci Rep. 2018;5(1):36-47.

40. Wickens MM, Bangasser DA, Briand LA. Sex differences in psychiatric disease: a focus on the glutamate system. Front Mol Neurosci. 2018;11:197.

41. Park L, et al. Neurophysiological changes associated with antidepressant response to ketamine not observed in a negative trial of scopolamine in major depressive disorder. Int J Neuropsychopharmacol. 2019;22(1):10-18.

42. Perova Z, Delevich K, Li B. Depression of excitatory synapses onto parvalbumin interneurons in the medial prefrontal cortex in susceptibility to stress. J Neurosci. 2015;35(7):3201-3206.

43. Murray AJ, et al. Parvalbumin-positive interneurons of the prefrontal cortex support working memory and cognitive flexibility. Sci Rep. 2015;5:16778.

44. Pozzi L, Pollak Dorocic I, Dorocic IP, Wang X, Carlén M, Meletis K. Mice lacking NMDA receptors in parvalbumin neurons display normal depression-related behavior and response to antidepressant action of NMDAR antagonists. PLoS One. 2014;9(1):e83879.

45. Fuchs T, Jefferson SJ, Hooper A, Yee PH, Maguire J, Luscher B. Disinhibition of somatostatin-positive GABAergic interneurons results in an anxiolytic and antidepressant-like brain state. $\mathrm{Mol}$ Psychiatry. 2017;22(6):920-930.

46. Ota KT, et al. REDD1 is essential for stressinduced synaptic loss and depressive behavior. Nat Med. 2014;20(5):531-535.

47. Duman RS, Sanacora G, Krystal JH. Altered connectivity in depression: GABA and Glutamate neurotransmitter deficits and reversal by novel treatments. Neuron. 2019;102(1):75-90.

48. Ghosal S, et al. Ketamine rapidly reverses stress-induced impairments in GABAergic transmission in the prefrontal cortex in male rodents [published online ahead of print November 7, 2019]. Neurobiol Dis. https://doi.org/10.1016/j. nbd.2019.104669.

49. Ng LHL, Huang Y, Han L, Chang RC, Chan YS, Lai CSW. Ketamine and selective activation of parvalbumin interneurons inhibit stress-induced dendritic spine elimination. Transl Psychiatry. 2018;8(1):272.

50. Taylor SR, Badurek S, Dileone RJ, Nashmi R, Minichiello L, Picciotto MR. GABAergic and glutamatergic efferents of the mouse ventral tegmental area. JComp Neurol. 2014;522(14):3308-3334.

51. Casanova E, et al. A CamKII $\alpha$ iCre BAC allows brain-specific gene inactivation. Genesis. 2001;31(1):37-42.

52. Brigman JL, et al. Loss of GluN2B-containing NMDA receptors in CA1 hippocampus and cortex impairs long-term depression, reduces dendritic spine density, and disrupts learning. J Neurosci. 2010;30(13):4590-4600.

53. Zhou FW, Chen HX, Roper SN. Balance of inhibitory and excitatory synaptic activity is altered in fast-spiking interneurons in experimental cortical dysplasia. J Neurophysiol. 2009;102(4):2514-2525.

54. Potapenko ES, Biancardi VC, Florschutz RM, Ryu PD, Stern JE. Inhibitory-excitatory synaptic balance is shifted toward increased excitation in magnocellular neurosecretory cells of heart failure rats. J Neurophysiol. 2011;106(3):1545-1557.

55. Budzillo A, Duffy A, Miller KE, Fairhall AL, Perkel DJ. Dopaminergic modulation of basal ganglia output through coupled excitation-inhibition. Proc Natl Acad Sci U S A. 2017;114(22):5713-5718.

56. Reynolds A, Leake D, Boese Q, Scaringe S, Marshall WS, Khvorova A. Rational siRNA design for RNA interference. Nat Biotechnol. 2004;22(3):326-330.

57. Ventura A, et al. Cre-lox-regulated conditional RNA interference from transgenes. Proc Natl Acad Sci U S A. 2004;101(28):10380-10385.

58. Konsman JP. The mouse brain in stereotaxic coordinates. Psychoneuroendocrinology. 2003;28(6):827-828.

59. Elsayed M, Banasr M, Duric V, Fournier NM, Licznerski P, Duman RS. Antidepressant effects of fibroblast growth factor-2 in behavioral and cellular models of depression. Biol Psychiatry. 2012;72(4):258-265.

60. Macbeth AH, Edds JS, Young WS. Housing conditions and stimulus females: a robust social discrimination task for studying male rodent social recognition. Nat Protoc. 2009;4(11):1574-1581. 